\title{
Synthesis of 2-\{[5-(aralkyl/aryl)-1,3,4-oxadiazol-2- yl]sulfanyl\}-N-(4-methyl-1,3-thiazol-2-yl)acetamides: Novel bi-heterocycles as potential therapeutic agents
}

\author{
Muhammad S Ramzan ${ }^{1}$, Muhammad A Abbasi ${ }^{1 \star}$, Aziz-ur-Rehman ${ }^{1}$, Sabahat Z \\ Siddiqui ${ }^{1}$, Syed AA Shah ${ }^{2,3}$, Muhammad Ashraf ${ }^{4}$, Muhammad A Lodhi ${ }^{5}$, Farman \\ A Khan ${ }^{5}$, Bushra Mirza ${ }^{6}$ \\ ${ }^{1}$ Department of Chemistry, Government College University, Lahore-54000, Pakistan, ${ }^{2}$ Faculty of Pharmacy, ${ }^{3}$ Atta-ur-Rahman \\ Institute for Natural Products Discovery (AuRIns), Level 9, FF3, Universiti Teknologi MARA, Puncak Alam Campus, 42300 \\ Bandar Puncak Alam, Selangor Darul Ehsan, Malaysia, ${ }^{4}$ Department of Chemistry, The Islamia University of Bahawalpur, \\ Bahawalpur-63100, ${ }^{5}$ Department of Biochemistry, Abdul Wali Khan University, Mardan-23200, ${ }^{6}$ Department of Biochemistry, \\ Quaid-i-Azam University, Islamabad, 45320, Pakistan
}

*For correspondence: Email: atrabbasi@yahoo.com; abbasi@gcu.edu.pk; Tel: (+92)-42-111000010 ext.266

\begin{abstract}
Purpose: To evaluate the therapeutic potential of new bi-heterocycles containing a 1,3-thiazole and 1,3,4-oxadiazole in the skeleton against Alzheimer's disease and diabetes, supported by in-silico study. Methods: The synthesis was initiated by the reaction of 4-methyl-1,3-thiazol-2-amine (1) with bromoacetyl bromide (2) in aqueous basic medium to obtain an electrophile,2-bromo-N-(4-methyl-1,3thiazol-2-yl)acetamide (3). In parallel reactions, a series of carboxylic acids, 4a-r, were converted through a sequence of three steps, into respective 1,3,4-oxadiazole heterocyclic cores, 7a-r, to utilize as nucleophiles. Finally, the designed molecules, 8a-r, were synthesized by coupling 7a-r individually with 3 in an aprotic polar solvent. The structures of these bi-heterocycles were elucidated by infrared (IR), electron ionization-mass spectrometry (EI-MS), proton nuclear magnetic resonance ( $\left.{ }^{1} \mathrm{H}-\mathrm{NMR}\right)$ and carbon nuclear magnetic resonance $\left({ }^{13} \mathrm{C}-\mathrm{NMR}\right)$. To evaluate their enzyme inhibitory potential, 8a-r were screened against acetylcholinesterase (AChE), but brine shrimp lethality bioassay.

Results: The most active compound against AChE was 81 with half-maximal inhibitory concentration $\left(I C_{50}\right)$ of $17.25 \pm 0.07 \mu \mathrm{M}$. Against BChE, the highest inhibitory effect was shown by $8 \boldsymbol{k}(56.23 \pm 0.09$ $\mu \mathrm{M})$. Compound $8 \mathrm{f}(161.26 \pm 0.23 \mu \mathrm{M})$ was recognized as a fairly good inhibitor of urease. In view of its inhibition of $\alpha$-glucosidase, $80(57.35 \pm 0.17 \mu \mathrm{M})$ was considered a potential therapeutic agent.

Conclusion: The results indicate that some of the synthesized products with low toxicity exhibit notable enzyme inhibitory activity against selected enzymes compared with the reference drug, and therefore, are of potential therapeutic interest
\end{abstract}

Keywords: 4-Methyl-1,3-thiazol-2-amine,1,3,4-Oxadiazole, Cholinesterases, a-Glucosidase, Urease, Brine shrimp

This is an Open Access article that uses a funding model which does not charge readers or their institutions for access and distributed under the terms of the Creative Commons Attribution License (http://creativecommons.org/licenses/by/4.0) and the Budapest Open Access Initiative (http://www.budapestopenaccessinitiative.org/read), which permit unrestricted use, distribution, and reproduction in any medium, provided the original work is properly credited.

Tropical Journal of Pharmaceutical Research is indexed by Science Citation Index (SciSearch), Scopus, International Pharmaceutical Abstract, Chemical Abstracts, Embase, Index Copernicus, EBSCO, African Index Medicus, JournalSeek, Journal Citation Reports/Science Edition, Directory of Open Access Journals (DOAJ), African Journal Online, Bioline International, Open-J-Gate and Pharmacy Abstracts 


\section{INTRODUCTION}

The chemistry and biological study of heterocyclic compounds has been an interesting field for a long time in medicinal chemistry. Thiazoles play a crucial role in the activity of biological compounds. For example, the thiazole ring is a component of vitamin B1 (thiamine), an important coenzyme of carboxylases. This heterocycle is also present in penicillins, which serve as antibiotics [1]. In addition, high antioxidant, anti-inflammatory and inhibitory effects of thiazoles have been observed in vitro in their action against parasites (Plasmodium and Trypanosoma) and fungi (Candida albicans) [2]. Similarly, 1,3,4-oxadiazoles have demonstrated a range of bioactivities [3-5].Molecules bearing this moiety are known to possess anti-parasitic, hypoglycemic, anti-inflammatory, antifungal and antibacterial activities [6-8].

Cholinesterases, acetylcholinesterase (AChE) and butyrylcholinesterase (BChE), belong to the class of serine hydrolases. Acetylcholine activity is terminated at cholinergic synapses by these enzymes, and these enzymes are also found at neuromuscular junctions and cholinergic brain synapses [9]. It is known that BChE is associated with Alzheimer's plaque in notably elevated quantities. The inhibitors of these enzymes may have future prospects as curative agents for Alzheimer's disease [10]. a-Glucosidase inhibitors (AGIs) such as acarbose and miglitol have been approved for clinical use in controlling the digestion of complex carbohydrates in the gut and may be used in the treatment of patients with type 2 diabetes or impaired glucose tolerance [11]. Urease is involved in different pathogenic processes such as pyelonephritis, peptic ulceration, hepatic encephalopathy, urolithiasis and urinary catheter encrustation [12]. Molecular docking analysis approximates ligands regarding their orientation and conformation at the binding site of target proteins. The precise prediction of activity and structural modeling can be achieved by docking studies. Furthermore, it elucidates the active site of proteins and interactions by inhibitors [13].

New drugs are continually being synthesized, aimed at treating and/or preventing various human diseases. The documented bioactivities of heterocyclic compounds prompted us to synthesize molecules having an amalgamation of two heterocyclic cores, thiazole and 1,3,4oxadiazole, linked through an acetamide. As it is known that structural modifications have an effect on the therapeutic behavior of drugs [14], the molecules were synthesized with variation of the groups attached to the 5-position of the 1,3,4- oxadiazole core. The whole series of compounds was tested against the four aforementioned enzymes and also cytotoxicity.

\section{EXPERIMENTAL}

\section{General}

All the chemicals and analytical grade solvents were purchased from Sigma Aldrich, Alfa Aesar (Germany) and Merck through local suppliers. Pre-coated silica gel Al-plates were used for TLC with ethyl acetate and $n$-hexane as solvent system. Spots were detected under UV light at $254 \mathrm{~nm}$. With open capillary tubes, a Gallonkamp apparatus was used to determine the melting point. IR spectra were recorded using the $\mathrm{KBr}$ pellet method on a Jasco-320-A spectrometer. ${ }^{1} \mathrm{H}-\mathrm{NMR}$ spectra were recorded at $600 \mathrm{MHz}$ in DMSO with a Bruker spectrometer. Mass spectra (EIMS) were obtained on a JEOL JMS-600H instrument with data system. The coupling constant $(\mathcal{J})$ is given in $\mathrm{Hz}$ and chemical shift $(\delta)$ in ppm. The abbreviations used in the interpretation of ${ }^{1} \mathrm{H}$ NMR spectra are as follows: $\mathrm{s}$, singlet; $\mathrm{d}$, doublet; dd, doublet of doublets; $\mathrm{t}$, triplet; br.t, broad triplet; q, quartet; quin, quintet; sex, sextet; sep, septet; m, multiplet.

Procedure for synthesis of 2-bromo-N-(4methyl-1,3-thiazol-2-yl)acetamide (3)

4-Methyl-1,3-thiazol-2-amine $(1 ; 0.038 \mathrm{~mol})$ was dissolved in $25 \mathrm{~mL}$ of distilled water in an iodine flask $(100 \mathrm{~mL})$ and $20 \%$ aq. $\mathrm{Na}_{2} \mathrm{CO}_{3}$ was added to adjust the $\mathrm{pH}$ to $9-10$. 2-Bromoethanoyl bromide (2; $0.038 \mathrm{~mol}$; bromoacetyl bromide) was added gradually with vigorous shaking and the mixture then allowed to stir for $2 \mathrm{~h}$. Reaction completion was monitored by TLC. Excess icecold distilled water $(40 \mathrm{~mL})$ was added, and the precipitate formed was collected by filtration. This precipitate of $\mathbf{3}$ was washed with distilled water and dried.

\section{General procedure for synthesis of ethyl aralkyl/arylcarboxylates (5a-r)}

Aralkyl/arylcarboxylic acids (4a-r; $2.5 \mathrm{~g}$ each) were refluxed, individually, with $60 \mathrm{~mL}$ of $\mathrm{EtOH}$ for 4-5 $\mathrm{h}$ in the presence of conc. $\mathrm{H}_{2} \mathrm{SO}_{4}(1.25$ $\mathrm{mL}$ ) in a $250-\mathrm{mL}$ round-bottom flask. TLC plates were used to round-bottom monitor the reactions. Excess distilled water $(150 \mathrm{~mL})$ was added after maximum completion of the reaction, and the $\mathrm{pH}$ was adjusted to 8-10 with $20 \%$ aq. $\mathrm{Na}_{2} \mathrm{CO}_{3}$. The product was collected through sequential extraction with $\mathrm{CHCl}_{3}(50 \mathrm{~mL} \times 3) . \mathrm{CHCl}_{3}$ was distilled off to collect the products. In some cases, the products were collected by filtration. 
The esters obtained, 5a-r, were then used further.

\section{General procedure for synthesis of aralkyl/arylcarbohydrazides (6a-r)}

Ethyl esters (5a-r; $4.5 \mathrm{~mL})$ were refluxed with $80 \% \mathrm{~N}_{2} \mathrm{H}_{4} \cdot \mathrm{H}_{2} \mathrm{O}(7.2 \mathrm{~mL})$ for $3-4 \mathrm{~h}$ in $20 \mathrm{~mL}$ of $\mathrm{EtOH}$ in a round-bottom flask $(100 \mathrm{~mL})$. The reaction was monitored by TLC. At completion, excess ice-cold distilled $\mathrm{H}_{2} \mathrm{O}(60 \mathrm{~mL})$ was added to obtain the precipitate, which was filtered off, washed with distilled $\mathrm{H}_{2} \mathrm{O}$ and dried to acquire the compounds, 6a-r.

General procedure for synthesis of 5aralkyl/aryl-1,3,4-oxadiazol-2-thiol (7a-r)

Solid $\mathrm{KOH}(0.029 \mathrm{~mol})$ was dissolved in $25 \mathrm{~mL}$ of $\mathrm{EtOH}$ on reflux in a $100-\mathrm{mL}$ round-bottomflask. Aralkyl/arylcarbohydrazides (6a-r; $0.029 \mathrm{~mol}$,), individually, were refluxed with $\mathrm{CS}_{2}(0.058 \mathrm{~mol})$ in this alkaline $\mathrm{EtOH}$ for $5-6 \mathrm{~h}$. Reaction was monitored by TLC. At completion, excess icecold distilled $\mathrm{H}_{2} \mathrm{O}(60 \mathrm{~mL})$ was added to form a homogeneous solution. $\mathrm{pH}$ was adjusted to 5-6 by adding dilute $\mathrm{HCl}$, and the precipitate formed was filtered off, washed with distilled $\mathrm{H}_{2} \mathrm{O}$ and dried. The products formed, 7a-r, were also recrystallized from EtOH.

General procedure for synthesis of $2-\{[5-$ aralkyl/aryl-1,3,4-oxadiazol-2-yl]sulfanyl\}- $\mathrm{N}$-(4methyl-1,3-thiazol-2-yl)acetamides (8a-r)

5-Aralkyl/aryl-1,3,4-oxadiazol-2-thiols

(7a-r; $0.004 \mathrm{~mol}$ ), individually, were dissolved in DMF $(11 \mathrm{~mL})$ in a $50-\mathrm{mL}$ round-bottom flask. $\mathrm{LiH}$ $(0.004 \mathrm{~mol})$ was then added, and the mixture was stirred for $0.5 \mathrm{~h}$. 2-Bromo- $\mathrm{N}$-(4-methyl-1,3thiazol-2-yl)acetamide (3; $0.004 \mathrm{~mol})$ was added and the mixture further stirred for 4-6 h. Reaction completion was confirmed by TLC. Excess icecold distilled water $(25 \mathrm{~mL})$ was then added gradually to the reaction mixture with continuous stirring. Aqueous $\mathrm{NaOH}$ was added dropwise with gentle shaking to adjust the $\mathrm{pH}$ to $8-10$. The reaction mixture was stirred for $1 \mathrm{~h}$, and the precipitates of 8a-r were filtered off, washed with distilled $\mathrm{H}_{2} \mathrm{O}$ and dried.

\section{Enzyme inhibition assays}

\section{Cholinesterase inhibition assay}

AChE and BChE inhibitory activity was determined in 96-well microplates according to a reported method [15] with slight modifications. The reaction mixture totaled $100 \mu \mathrm{L}$ and contained the following: $60 \mu \mathrm{L}$ of $50 \mathrm{mM}$
$\mathrm{Na}_{2} \mathrm{HPO}_{4}$ buffer, $\mathrm{pH} \quad 7.7 ; \quad 10 \mu \mathrm{L}$ of acetylthiocholine iodide for AChE and butyrylthiocholine bromide for $\mathrm{BChE}$, at final concentrations of acetylthiocholine iodide; $10 \mu \mathrm{L}$ of test compound at $0.5 \mathrm{mM}$, and $10 \mu \mathrm{L}$ of enzyme (0.005 U/well AChE or BChE). The microplates were mixed, pre-read, and incubated for $10 \mathrm{~min}$ at $37^{\circ} \mathrm{C}$. The enzyme reaction product was determined by the addition of $0.5 \mathrm{mM}$ Ellman's reagent (5,5'-dithio-bis-[2-nitrobenzoic acid)and further incubation for $15 \mathrm{~min}$ at $37^{\circ} \mathrm{C}$, followed by reading absorbance at $405 \mathrm{~nm}$. All experiments were performed with controls and in triplicate. Eserine $(0.5 \mathrm{mM})$ served as the positive control. Inhibition $(\mathrm{H})$ was calculated using Eq 1.

$H(\%)=\{(A c-A t) / A c\} 100$

where $A c=$ absorbance of control and At $=$ absorbance of test compound.

$\mathrm{IC}_{50}$ values were calculated using EZ-Fit Enzyme kinetics software (Perrella Scientific Inc. Amherst, $\mathrm{NH}$, USA).

\section{a-Glucosidase inhibition assay}

This enzyme inhibition assay was carried out in 96-well as previously reported [16], with modifications, in which a $100-\mu \mathrm{L}$ reaction mixture contained $70 \mu \mathrm{L}$ of phosphate-buffered saline, $\mathrm{pH} 6.8,10 \mu \mathrm{L}$ of test compound and 10 $\mu \mathrm{L}$ of enzyme ( $p$-nitrophenyl-alpha-Dglucopyranoside U/well).

The reaction mixture was mixed, incubated for $10 \mathrm{~min}$ at $37^{\circ} \mathrm{C}$ and pre-read at $400 \mathrm{~nm}$. The reaction was initiated by the addition of $10 \mu \mathrm{L}$ of $0.5 \mathrm{mM} p$-nitrophenyl-alpha-D-glucopyranoside. Acarbose was used as positive control. Microplates were incubated for $30 \mathrm{~min}$ at $37 \stackrel{\circ}{\circ} \mathrm{C}$ and absorbance read at $400 \mathrm{~nm}$ using a Synergy HT microplate reader. All experiments were performed in duplicate. The equation discussed for cholinesterase enzymes was used for the determination of $\%$ inhibition and $\mathrm{IC}_{50}$ values.

\section{Urease inhibition assay}

This enzyme inhibition assay is the customized form of the commonly known Berthelot assay [17]. To each well of a 96-well microplate, $45 \mu \mathrm{L}$ of reaction mixture containing $10 \mu \mathrm{L}$ of phosphate buffer, $\mathrm{pH} 7.0,10 \mu \mathrm{L}$ of test sample and $25 \mu \mathrm{L}$ of urease solution $(0.135 \mathrm{U})$ were added. Contents were pre-incubated at $37 \stackrel{\circ}{\circ} \mathrm{C}$ for $5 \mathrm{~min}$. A volume $(40 \mu \mathrm{L})$ of urea stock solution $(20 \mathrm{mM})$ was added to each well, and the microplate incubated for 10 $\min$ at $37^{\circ} \mathrm{C}$. This was followed by the addition of 
$115 \mu \mathrm{L}$ of phenol-hypochlorite reagent (freshly prepared by mixing $45 \mu \mathrm{L}$ phenol with $70 \mu \mathrm{L}$ of alkali) per well. For color development, the microplate was incubated for another $10 \mathrm{~min}$ at $37 \stackrel{\circ}{\circ}$, and absorbance measured at $625 \mathrm{~nm}$. The percentage enzyme inhibition and $I_{50}$ values were determined as mentioned above.

\section{Cytotoxicity assay}

Cytotoxicity was evaluated by the brine shrimp lethality bioassay [18]. Artificial sea water was prepared with sea salt at $34 \mathrm{~g} / \mathrm{L}$.A shallow rectangular dish $(22 \times 32 \mathrm{~cm})$ was used for the hatching of brine shrimp (Artemia salina) eggs (Sera, Heidelberg, Germany) under constant aeration for $48 \mathrm{~h}$ at room temperature. After hatching, active shrimp were collected from the brighter portion of the hatching chamber and used for the assay. Vials containing $5 \mathrm{~mL}$ of artificial sea water (with different concentrations of test compounds from the stock) were used. Ten shrimp were transferred to each vial. The temperature of the vials was maintained at 26 ${ }^{\circ} \mathrm{C}$. The number of surviving shrimp were calculated after one day. The experiment was performed in triplicate and data analyzed with the Finney computer program to determine the $\mathrm{LD}_{50}$ (lethal dose that killed $50 \%$ of shrimp).

\section{Molecular docking studies}

The reported MOE-Dock method of MOE 20092010 was utilized to study molecular recognition [19]. The Protein Data Bank site was used to retrieve protein molecules of acetylcholinesterase (PDB code: 1GQR), butyrylcholinesterase (PDB code: 1POP), $\alpha-$ glucosidase (PDB ID: $3 \mathrm{NO}_{4}$ ) and urease (PDB ID: 4UBP). After removing water molecules, MOE applications were used for performing 3D protonation of the protein molecule. The energy minimization algorithm of the MOE tool was used to minimize the energy of protein molecules using the following parameters; gradient of 0.05 , Force Field of MMFF94X \& Solvation and Chiral Constraint of Current Geometry. However, minimization of energy was ended for the gradient below 0.05 . The templates for docking were the energy minimized protonated structures that were saved in a separate database (using a mdb file format). Finally, all compounds were docked into the binding pockets of enzymes. For the confirmation of validity, a re-docking procedure was applied. After docking analysis of each compound with 30 conformations, the best 2D images were selected for their specific types of interactions and their 3D images were drawn along with their bond lengths.

\section{Statistical analysis}

All measurements were carried out in triplicate and statistical analysis was performed by Microsoft Excel 2010. The results are presented as mean \pm SEM with $90 \% \mathrm{CL}$. $P<0.05$ was considered statistically significant.

\section{RESULTS AND DISCUSSION}

\section{Chemistry}

The protocol for the synthesis of the new molecules, 2-\{[5-(aralkyl/aryl)-1,3,4-oxadiazol-2yl]sulfanyl\}- $N$-(4-methyl-1,3-thiazol-2-yl)

acetamides (8a-r), is sketched in Figure 1, and the various groups are given in Table 1 . The first step in the synthesis was the reaction of 4methyl-1,3-thiazol-2-amine (1) with 2bromoethanoyl bromide (2) in a basic aqueous medium to synthesize 2-bromo- $\mathrm{N}$-(4-methyl-1,3thiazol-2-yl)acetamide (3), an electrophile. Various nucleophiles were then synthesized in a parallel sequence of reactions, starting from a series of aralkyl/arylcarboxylic acids, 4a-r, which were converted to respective esters, 5a-r; acid hydrazides, 6a-r, which were cyclized to the respective 5-aralkyl/aryl-1,3,4-oxadiazole-2-thiols (7a-r). These thiols, serving as nucleophiles, were finally coupled one by one with 3in an aprotic polar solvent, i.e., DMF, using $\mathrm{LiH}$ as a base to obtain the target heterocyclic derivatives, 8a-r. The structural verification was performed through spectral data analysis. All compounds were tested for enzyme inhibitory activity against cholinesterases, urease, and a-glucosidase, and the results are as shown in Table 2. The brine shrimp lethality bioassay was used to evaluate the cytotoxicity of the synthesized compounds and these results are given in Table 3 .

\section{Spectral characteristics of the synthesized molecules}

\section{N-(4-Methyl-1,3-thiazol-2-yl)-2-[(5-phenyl- 1,3,4-oxadiazol-2-yl)sulfanyl]acetamide (8a)}

Dull-white solid; yield: $80 \%$; m.p.: $176-177{ }^{\circ} \mathrm{C}$ Mol. Formula: $\mathrm{C}_{14} \mathrm{H}_{12} \mathrm{~N}_{4} \mathrm{O}_{2} \mathrm{~S}_{2}$; Mol. Mass: 332 $\mathrm{g} / \mathrm{mol}$; IR $\left(\mathrm{KBr}, \mathrm{cm}^{-1}\right) v_{\text {max }}: 3347$ (N-H stretching), 2976 (C-H stretching of aromatic ring), 1678 ( $\mathrm{C}=\mathrm{N}$ stretching), $1644 \quad(\mathrm{C}=\mathrm{O}$ stretching), 1570 ( $\mathrm{C}=\mathrm{C}$ stretching of aromatic ring), 1154 (C-O-C stretching); ${ }^{1} \mathrm{H}-\mathrm{NMR} \quad$ (DMSO-d $6,600 \mathrm{MHz}$, $\delta / \mathrm{ppm}$ ): $\delta 12.47$ (s, 1H, CON-H), 7.93 (d, J = 8.5 $\mathrm{Hz}, 2 \mathrm{H}, \mathrm{H}-2$ '" \& H-6"'), 7.62-7.57 (m, 3H, H-3"' to H-5'"), 6.80 (br.s, $1 \mathrm{H}, \mathrm{H}-5$ ), 4.38 (br.s, $2 \mathrm{H}, \mathrm{CH}_{2^{-}}$ $\left.2^{\prime}\right), 2.27\left(\mathrm{~s}, 3 \mathrm{H}, \mathrm{CH}_{3}-6\right) ;{ }^{13} \mathrm{C}-\mathrm{NMR}$ (DMSO-d 6 , 150 $\mathrm{MHz}, \delta / \mathrm{ppm}): \delta 165.76$ (C-1'), 165.26 (C-5"), 162.98 (C-2"), 156.86 (C-2), 146.88 (C-4), 
<smiles>Cc1csc(N)n1</smiles>

4-Methyl-1,3-thiazol-2-amine<smiles>[R]c1nnc(SCC(=O)Nc2nc(C)cs2)o1</smiles>

2-[(5-Aralkyl/aryl-1,3,4-oxadiazol-2-yl)sulfanyl]$N$-(4-methyl-1,3-thiazol-2-yl)acetamides<smiles>Cc1csc(NC(=O)CBr)n1</smiles>

2-Bromo- $N$-(4-methyl-1,3-thiazol-2yl)acetamide

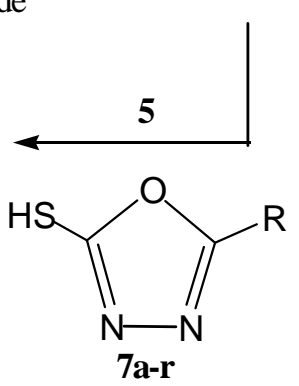

5-Aralkyl/aryl-1,3,4-oxadiazole-2-thiol<smiles>[R]C(=O)OC#CC(=O)OCC</smiles><smiles>C#CC(=O)NN</smiles>

Figure 1: Protocol for synthesis of 2-\{[5-(aralkyl/aryl)-1,3,4-oxadiazol-2-yl]sulfanyl\}-N-(4-methyl-1,3-thiazol-2yl)acetamides

132.06 (C-4"'), 129.38 (C-3"' \& C-5"'), 126.36 (C2"' \& C-6"'), 122.89 (C-1"'), 108.12 (C-5), 35.37 (C-2'), 16.83 (C-6); El-MS: m/z $332\left[\mathrm{M}^{+}, 219\right.$ $\left[\mathrm{C}_{10} \mathrm{H}_{7} \mathrm{~N}_{2} \mathrm{O}_{2} \mathrm{~S}\right]^{+}, \quad 192 \quad\left[\mathrm{C}_{9} \mathrm{H}_{7} \mathrm{~N}_{2} \mathrm{OS}\right]^{+}, \quad 114$ $\left[\mathrm{C}_{3} \mathrm{H}_{2} \mathrm{~N}_{2} \mathrm{OS}\right]^{+}, 77\left[\mathrm{C}_{6} \mathrm{H}_{5}\right]^{+}$.

\section{2-\{[5-(4-Methylphenyl)-1,3,4-oxadiazol-2- yl]sulfanyl\}-N-(4-methyl-1,3-thiazol-2- yl)acetamide (8b)}

Light-brown solid; yield: $79 \%$; m.p.: $227-228{ }^{\circ} \mathrm{C}$; Mol. Formula: $\mathrm{C}_{15} \mathrm{H}_{14} \mathrm{~N}_{4} \mathrm{O}_{2} \mathrm{~S}_{2}$; Mol. Mass: 346 $\mathrm{g} / \mathrm{mol}$; IR $\left(\mathrm{KBr}, \mathrm{cm}^{-1}\right) v_{\text {max }}: 3358$ (N-H stretching), 2977 (C-H stretching of aromatic ring), 1675 ( $\mathrm{C}=\mathrm{N}$ stretching), 1645 ( $\mathrm{C}=\mathrm{O}$ stretching), 1576 ( $\mathrm{C}=\mathrm{C}$ stretching of aromatic ring), 1169 (C-O-C stretching); ${ }^{1} \mathrm{H}-\mathrm{NMR}$ (DMSO-d $6,600 \mathrm{MHz}$, $\delta / \mathrm{ppm}$ ): $\delta 12.46$ (s, $1 \mathrm{H}, \mathrm{CON}-\mathrm{H}), 7.82$ (br.d, $J=$ $8.1 \mathrm{~Hz}, 2 \mathrm{H}, \mathrm{H}-2$ "' \& H-6"'), 7.38 (br.d, $J=7.8 \mathrm{~Hz}$, $2 \mathrm{H}, \mathrm{H}-3$ "' \& H-5"'), 6.79 (br.s, $1 \mathrm{H}, \mathrm{H}-5$ ), 4.37 (br.s, 2H, $\left.\mathrm{CH}_{2}-2^{\prime}\right), 2.38$ (s, 3H, $\left.\mathrm{CH}_{3}-7^{\prime \prime \prime}\right), 2.27$ (s, $\left.3 \mathrm{H}, \mathrm{CH}_{3}-6\right) ;{ }^{13} \mathrm{C}-\mathrm{NMR}$ (DMSO-d $6,150 \mathrm{MHz}$, $\delta / \mathrm{ppm}): \delta 165.35$ (C-1'), 164.35 (C-5"), 162.60 (C-2"), 156.7 (C-2), 142.25 (C-4), 141.0 (C-4"'), 129.92 (C-2"' \& C-6"'), 126.32 (C-3"' \& C-5"'), 120.15 (C-1"'), 108.06 (C-5), 35.41 (C-2'), 21.09 (C-7"'), 11.07 (C-6) ; El-MS: m/z 346 [M] ${ }^{+}, 233$ $\left[\mathrm{C}_{11} \mathrm{H}_{9} \mathrm{~N}_{2} \mathrm{O}_{2} \mathrm{~S}\right]^{+}, \quad 206 \quad\left[\mathrm{C}_{10} \mathrm{H}_{9} \mathrm{~N}_{2} \mathrm{OS}\right]^{+}, \quad 192$
$\left[\mathrm{C}_{8} \mathrm{H}_{5} \mathrm{~N}_{3} \mathrm{OS}\right]^{+}, 159\left[\mathrm{C}_{9} \mathrm{H}_{7} \mathrm{~N}_{2} \mathrm{O}\right]^{+}, 141\left[\mathrm{C}_{5} \mathrm{H}_{5} \mathrm{~N}_{2} \mathrm{OS}\right]^{+}$, $119\left[\mathrm{C}_{8} \mathrm{H}_{7} \mathrm{O}\right]^{+}, 104\left[\mathrm{C}_{7} \mathrm{H}_{4} \mathrm{O}\right]^{+}, 91\left[\mathrm{C}_{7} \mathrm{H}_{7}\right]^{+}, 65$ $\left[\mathrm{C}_{5} \mathrm{H}_{5}\right]^{+}$.

\section{2-\{[5-(4-Hydroxyphenyl)-1,3,4-oxadiazol-2- yl]sulfanyl\}-N-(4-methyl-1,3-thiazol-2- yl)acetamide (8c)}

Dull-white solid; yield: $80 \%$; m.p.: $186-187{ }^{\circ} \mathrm{C}$; Mol. Formula: $\mathrm{C}_{14} \mathrm{H}_{12} \mathrm{~N}_{4} \mathrm{O}_{3} \mathrm{~S}_{2}$; Mol. Mass: 348 $\mathrm{g} / \mathrm{mol}$; IR (KBr, $\left.\mathrm{cm}^{-1}\right) v_{\max }: 3356$ (N-H stretching), 2979 (C-H stretching of aromatic ring), 1667 ( $\mathrm{C}=\mathrm{N}$ stretching), $1646 \quad(\mathrm{C}=\mathrm{O}$ stretching $), 1571$ ( $\mathrm{C}=\mathrm{C}$ stretching of aromatic ring), 1148 (C-O-C stretching); ${ }^{1} \mathrm{H}-\mathrm{NMR} \quad$ (DMSO-d $6,600 \mathrm{MHz}$, $\delta / \mathrm{ppm}$ ): $\delta 12.43$ (s, $1 \mathrm{H}, \mathrm{CON}-\mathrm{H}), 7.96$ (br.d, $J=$ $8.5 \mathrm{~Hz}, 2 \mathrm{H}, \mathrm{H}-3^{\prime \prime \prime}$ \& $\mathrm{H}-5$ "'), 7.91 (br.d, $J=8.5 \mathrm{~Hz}$, $1 \mathrm{H}, \mathrm{H}-2$ "' \& H-6"'), 6.79 (s, 1H, H-5), 4.30 (br.s, $\left.2 \mathrm{H}, \quad \mathrm{CH}_{2}-2^{\prime}\right), 2.27$ (s, 3H, $\left.\mathrm{CH}_{3}-6\right) ;{ }^{13} \mathrm{C}-\mathrm{NMR}$ (DMSO-d $6,150 \mathrm{MHz}, \delta / \mathrm{ppm}): \delta 165.15$ (C-1'), 163.70 (C-5"), 163.32 (C-2"), 157.65 (C-2), 146.81 (C-4), 137.81 (C-3"' \& C-5"'), 133.28 (C4"'), 131.14 (C-2"' \& C-6"'), 122.07 (C-1"'), 108.05 (C-5), 35.38 (C-2'), 16.83 (C-6); El-MS:

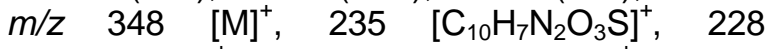
$\left[\mathrm{C}_{7} \mathrm{H}_{7} \mathrm{~N}_{3} \mathrm{O}_{2} \mathrm{~S}_{2}\right]^{+}, \quad 208 \quad\left[\mathrm{C}_{9} \mathrm{H}_{8} \mathrm{~N}_{2} \mathrm{O}_{2} \mathrm{~S}\right]^{+}, \quad 175$ $\left[\mathrm{C}_{9} \mathrm{H}_{7} \mathrm{~N}_{2} \mathrm{O}_{2}\right]^{+}, 161\left[\mathrm{C}_{9} \mathrm{H}_{9} \mathrm{~N}_{2} \mathrm{O}\right]^{+}, 141\left[\mathrm{C}_{5} \mathrm{H}_{5} \mathrm{~N}_{2} \mathrm{OS}\right]^{+}$, $121\left[\mathrm{C}_{7} \mathrm{H}_{5} \mathrm{O}_{2}\right]^{+}, 114\left[\mathrm{C}_{4} \mathrm{H}_{5} \mathrm{~N}_{2} \mathrm{~S}\right]^{+}, 93\left[\mathrm{C}_{6} \mathrm{H}_{5} \mathrm{O}\right]^{+}$. 
Table 1: Different aralkyl/aryl groups in 2-\{[5-(aralkyl/aryl)-1,3,4-oxadiazol-2-yl]sulfanyl\}- N-(4-methyl-1,3-thiazol2-yl)acetamides (8a-r)

\begin{tabular}{|c|c|c|c|c|c|}
\hline Comp. & $\mathbf{R}$ & Comp. & $\mathbf{R}$ & Comp. & $\mathbf{R}$ \\
\hline $8 a$ & & $8 g$ & & $8 m$ & \\
\hline $8 b$ & & $8 \mathrm{~h}$ & & $8 n$ & \\
\hline $8 c$ & & $8 i$ & & 80 & \\
\hline $8 d$ & & $8 j$ & & $8 p$ & \\
\hline $8 e$ & & $8 k$ & & $8 q$ & \\
\hline $8 f$ & & 81 & & $8 r$ & \\
\hline
\end{tabular}

Protocol for synthesis of 2-\{[5-(aralkyl/aryl)-1,3,4-oxadiazol-2-yl]sulfanyl\}- $N$-(4-methyl-1,3-thiazol-2-yl)acetamides (8a-r). Reagents \& conditions: (1) $\mathrm{H}_{2} \mathrm{O}, 20 \% \mathrm{Na}_{2} \mathrm{CO}_{3}$, stirring for $2 \mathrm{~h}$. (2) $\mathrm{H}_{2} \mathrm{SO}_{4}$, EtOH, refluxing for 4-5 h. (3) $\mathrm{N}_{2} \mathrm{H}_{4}, \mathrm{EtOH}$, refluxing for $3-4 \mathrm{~h}$. (4) $\mathrm{CS}_{2}, \mathrm{KOH}, \mathrm{EtOH}$, refluxing for $5-6 \mathrm{~h}$. (5) DMF, LiH, stirring for 4-6 h

\section{2-\{[5-(2-Methoxyphenyl)-1,3,4-oxadiazol-2- yl]sulfanyl\}-N-(4-methyl-1,3-thiazol-2- yl)acetamide (8d)}

Light-brown solid; yield: $75 \%$; m.p.: $157-158{ }^{\circ} \mathrm{C}$; Mol. Formula: $\mathrm{C}_{15} \mathrm{H}_{14} \mathrm{~N}_{4} \mathrm{O}_{3} \mathrm{~S}_{2}$; Mol. Mass: 362 $\mathrm{g} / \mathrm{mol}$; IR $\left(\mathrm{KBr}, \mathrm{cm}^{-1}\right) v_{\text {max }}: 3358$ (N-H stretching), 2977 (C-H stretching of aromatic ring), 1674 ( $\mathrm{C}=\mathrm{N}$ stretching), $1644 \quad(\mathrm{C}=\mathrm{O}$ stretching), 1575 ( $\mathrm{C}=\mathrm{C}$ stretching of aromatic ring), 1163 (C-O-C stretching); ${ }^{1} \mathrm{H}-\mathrm{NMR} \quad$ (DMSO-d $6,600 \mathrm{MHz}$, $\delta / \mathrm{ppm}): \delta 12.46(\mathrm{~s}, 1 \mathrm{H}, \mathrm{CON}-\mathrm{H}), 7.76(\mathrm{dd}, J=$ $1.5,7.6 \mathrm{~Hz}, 1 \mathrm{H}, \mathrm{H}-6 " '), 7.60$ (dt, $J=1.5,8.6 \mathrm{~Hz}$, $\left.1 \mathrm{H}, \mathrm{H}-5^{\prime \prime \prime}\right), 7.24$ (br.d, $\left.J=8.4 \mathrm{~Hz}, 1 \mathrm{H}, \mathrm{H}-3^{\prime \prime \prime}\right), 7.09$ (br.t, $J=7.5 \mathrm{~Hz}, 1 \mathrm{H}, \mathrm{H}-4$ '"), $6.80(\mathrm{~s}, 1 \mathrm{H}, \mathrm{H}-5)$, 4.38 (br.s, $2 \mathrm{H}, \mathrm{CH}_{2}-2$ '), 3.85 (br.s, $\left.3 \mathrm{H}, \mathrm{CH}_{3} \mathrm{O}-7{ }^{\prime \prime \prime}\right)$, 2.27 (s, 3H, $\left.\mathrm{CH}_{3}-6\right) ;{ }^{13} \mathrm{C}-\mathrm{NMR}$ (DMSO-d 6,150 $\mathrm{MHz}, \delta / \mathrm{ppm}): \delta 165.15$ (C-1'), 164.0 (C-5"), 162.71 (C-2"), 157.86 (C-2"'), 156.92 (C-2), 146.85 (C-4), 133.60 (C-5"'), 133.01 (C-6"'), 120.68 (C-4"'), 112.62 (C-3"'), 111.79 (C-1"'),
108.06 (C-5), 55.92 (C-7"'), 35.34 (C-2'), 16.81 (C-6); El-MS: $m / z 362[\mathrm{M}]^{+}, 257\left[\mathrm{C}_{8} \mathrm{H}_{9} \mathrm{~N}_{4} \mathrm{O}_{2} \mathrm{~S}_{2}\right]^{+}$, $249\left[\mathrm{C}_{11} \mathrm{H}_{9} \mathrm{~N}_{2} \mathrm{O}_{3} \mathrm{~S}\right]^{+}, 222\left[\mathrm{C}_{10} \mathrm{H}_{9} \mathrm{~N}_{2} \mathrm{O}_{2} \mathrm{~S}\right]^{+}, 189$ $\left[\mathrm{C}_{6} \mathrm{H}_{9} \mathrm{~N}_{2} \mathrm{OS}_{2}\right]^{+}, 175\left[\mathrm{C}_{9} \mathrm{H}_{7} \mathrm{~N}_{2} \mathrm{O}_{2}\right]^{+}, 161\left[\mathrm{C}_{9} \mathrm{H}_{9} \mathrm{~N}_{2} \mathrm{O}\right]^{+}$, $141 \quad\left[\mathrm{C}_{5} \mathrm{H}_{5} \mathrm{~N}_{2} \mathrm{OS}\right]^{+}, \quad{ }_{135} \quad\left[\mathrm{C}_{8} \mathrm{H}_{7} \mathrm{O}\right]^{+}, \quad 114$ $\left[\mathrm{C}_{3} \mathrm{H}_{2} \mathrm{~N}_{2} \mathrm{OS}\right]^{+}, 91\left[\mathrm{C}_{7} \mathrm{H}_{8}\right]^{+}, 77\left[\mathrm{C}_{6} \mathrm{H}_{5}\right]^{+}$.

\section{2-\{[5-(2-Chlorophenyl)-1,3,4-oxadiazol-2- yl]sulfanyl\}-N-(4-methyl-1,3-thiazol-2- yl)acetamide (8e)}

Light-brown solid; yield: $77 \%$; m.p.: $158-159{ }^{\circ} \mathrm{C}$; Mol. Formula: $\mathrm{C}_{14} \mathrm{H}_{11} \mathrm{ClN}_{4} \mathrm{O}_{2} \mathrm{~S}_{2}$; Mol. Mass: 366 $\mathrm{g} / \mathrm{mol}$; IR $\left(\mathrm{KBr}, \mathrm{cm}^{-1}\right) v_{\max }: 3350$ (N-H stretching), 2973 (C-H stretching of aromatic ring), 1670 ( $\mathrm{C}=\mathrm{N}$ stretching), $1640 \quad(\mathrm{C}=\mathrm{O}$ stretching), 1572 ( $\mathrm{C}=\mathrm{C}$ stretching of aromatic ring), 1159 (C-O-C stretching), 684 (C-Cl stretching); ${ }^{1} \mathrm{H}-\mathrm{NMR}$ (DMSO-d $6,600 \mathrm{MHz}, \delta / \mathrm{ppm}): \delta 12.47(\mathrm{~s}, 1 \mathrm{H}$, CON-H), 7.94 (dd, $J=1.6,7.8 \mathrm{~Hz}, 1 \mathrm{H}, \mathrm{H}-3^{\prime \prime \prime}$ ), 7.69 (br.d, $\left.J=7.9 \mathrm{~Hz}, 1 \mathrm{H}, \mathrm{H}-6{ }^{\prime \prime \prime}\right), 7.62$ (dt, $J=$ 
1.6, 8.1 Hz, 1H, H-4"'), 7.53 (dt, $J=0.9,6.6 \mathrm{~Hz}$, $1 \mathrm{H}, \mathrm{H}-5$ "'), 6.80 (br.s, 1H, H-5), 4.40 (br.s, 2H, $\mathrm{CH}_{2}-2^{\prime}$ ), 2.27 (s, 3H, $\left.\mathrm{CH}_{3}-6\right)$; ${ }^{13} \mathrm{C}-\mathrm{NMR}$ (DMSO$\left.\mathrm{d}_{6}, 150 \mathrm{MHz}, \delta / \mathrm{ppm}\right): \delta 165.01$ (C-1'), 163.39 (C5"), 163.30 (C-2"), 156.66 (C-2), 146.87 (C-4), 133.30 (C-4"'), 131.66 (C-2"'), 131.14 (C-3"'), 131.10 (C-6"'), 127.82 (C-5"'), 122.06 (C-1"'), 108.12 (C-5), 35.39 (C-2'), 16.84 (C-6); El-MS: $\mathrm{m} / \mathrm{z} 368[\mathrm{M}+2]^{+}, 366[\mathrm{M}]^{+}, 253\left[\mathrm{C}_{10} \mathrm{H}_{6} \mathrm{ClN}_{2} \mathrm{O}_{2} \mathrm{~S}\right]^{+}$, $226\left[\mathrm{C}_{9} \mathrm{H}_{6} \mathrm{CIN}_{2} \mathrm{OS}\right]^{+}, \quad 193 \quad\left[\mathrm{C}_{8} \mathrm{H}_{6} \mathrm{~N}_{3} \mathrm{OS}\right]^{+}, 179$ $\left[\mathrm{C}_{8} \mathrm{H}_{4} \mathrm{CIN} \mathrm{OS}^{+}, 139\left[\mathrm{C}_{5} \mathrm{H}_{3} \mathrm{~N}_{2} \mathrm{OS}\right]^{+}, 125\left[\mathrm{C}_{7} \mathrm{H}_{5} \mathrm{Cl}\right]^{+}\right.$, $114\left[\mathrm{C}_{3} \mathrm{H}_{2} \mathrm{~N}_{2} \mathrm{OS}\right]^{+}, 75\left[\mathrm{C}_{6} \mathrm{H}_{3}\right]^{+}$.

\section{2-\{[5-(3-Chlorophenyl)-1,3,4-oxadiazol-2- yl]sulfanyl\}-N-(4-methyl-1,3-thiazol-2- yl)acetamide (8f)}

Light-brown solid; yield: $79 \%$; m.p.: $189-190{ }^{\circ} \mathrm{C}$; Mol. Formula: $\mathrm{C}_{14} \mathrm{H}_{11} \mathrm{ClN}_{4} \mathrm{O}_{2} \mathrm{~S}_{2}$; Mol. Mass: 366 $\mathrm{g} / \mathrm{mol}$; IR ( $\left.\mathrm{KBr}, \mathrm{cm}^{-1}\right) v_{\text {max }}: 3368$ (N-H stretching), 2979 (C-H stretching of aromatic ring), 1674 ( $\mathrm{C}=\mathrm{N}$ stretching), 1646 ( $\mathrm{C}=\mathrm{O}$ stretching), 1571 $(\mathrm{C}=\mathrm{C}$ stretching of aromatic ring), 1155 (C-O-C stretching), 689 (C-Cl stretching); ${ }^{1} \mathrm{H}-\mathrm{NMR}$ (DMSO-d $6,600 \mathrm{MHz}, \delta / \mathrm{ppm}): \delta 12.47$ (s, $1 \mathrm{H}$, CON-H), 7.98 (dd, $J=2.1,8.5 \mathrm{~Hz}, 1 \mathrm{H}, \mathrm{H}-6^{\prime \prime \prime}$ ), 7.96 (br.t, $\left.J=8.5 \mathrm{~Hz}, 1 \mathrm{H}, \mathrm{H}-5^{\prime \prime \prime)}\right), 7.52$ (d, $J=2.1$ Hz, 1H, H-2"'), 7.49 (dd, J = 2.1, 8.5 Hz, 1H, H4"'), 6.80 (br.s, 1H, H-5), 4.40 (br.s, $2 \mathrm{H}, \mathrm{CH}_{2}-2$ '), 2.27 (s, 3H, $\left.\mathrm{CH}_{3}-6\right) ;{ }^{13} \mathrm{C}-\mathrm{NMR}$ (DMSO-d 6,150 $\mathrm{MHz}, \delta / \mathrm{ppm}): \delta 165.15$ (C-1'), 163.70 (C-5"), 163.32 (C-2"), 157.65 (C-2), 146.81 (C-4), 137.81 (C-3"'), 133.28 (C-4"'), 131.14 (C-2"'), 131.09 (C-6"'), 127.82 (C-5"'), 122.07 (C-1"'), 108.12 (C-5), 35.38 (C-2'), 16.84 (C-6); El-MS:

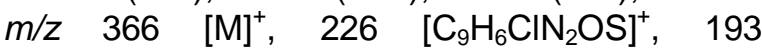
$\left[\mathrm{C}_{8} \mathrm{H}_{6} \mathrm{~N}_{3} \mathrm{OS}\right]^{+}, \quad 179 \quad\left[\mathrm{C}_{8} \mathrm{H}_{4} \mathrm{CIN}_{2} \mathrm{OS}\right]^{+}, \quad 139$ $\left[\mathrm{C}_{5} \mathrm{H}_{3} \mathrm{~N}_{2} \mathrm{OS}\right]^{+}, 125\left[\mathrm{C}_{7} \mathrm{H}_{5} \mathrm{Cl}\right]^{+}, 114\left[\mathrm{C}_{3} \mathrm{H}_{2} \mathrm{~N}_{2} \mathrm{OS}\right]^{+}$, $75\left[\mathrm{C}_{6} \mathrm{H}_{3}\right]^{+}$.

\section{2-\{[5-(4-Chlorophenyl)-1,3,4-oxadiazol-2- yl]sulfanyl\}-N-(4-methyl-1,3-thiazol-2- yl)acetamide (8g)}

Light-brown solid; yield: $76 \%$; m.p.: $187-188{ }^{\circ} \mathrm{C}$; Mol. Formula: $\mathrm{C}_{14} \mathrm{H}_{11} \mathrm{CIN}_{4} \mathrm{O}_{2} \mathrm{~S}_{2}$; Mol. Mass: 366 $\mathrm{g} / \mathrm{mol}$; IR $\left(\mathrm{KBr}, \mathrm{cm}^{-1}\right) v_{\text {max }}: 3347$ (N-H stretching), 2979 (C-H stretching of aromatic ring), 1681 ( $\mathrm{C}=\mathrm{N}$ stretching), 1636 ( $\mathrm{C}=\mathrm{O}$ stretching), 1584 $(\mathrm{C}=\mathrm{C}$ stretching of aromatic ring), 1165 (C-O-C stretching), 691 (C-Cl stretching); ${ }^{1} \mathrm{H}-\mathrm{NMR}$ (DMSO-d $6,600 \mathrm{MHz}, \delta / \mathrm{ppm}): \delta 12.47$ (s, 1H, CON-H), 7.91 (br.d, $J=8.5 \mathrm{~Hz}, 2 \mathrm{H}, \mathrm{H}-2$ "' \& H6"'), 7.49 (br.d, $J=8.5 \mathrm{~Hz}, 1 \mathrm{H}, \mathrm{H}-3$ "' \& H-5"'), 6.80 (br.s, $1 \mathrm{H}, \mathrm{H}-5$ ), 4.40 (br.s, $2 \mathrm{H}, \mathrm{CH}_{2}-2$ '), 2.27 (s, $\left.3 \mathrm{H}, \mathrm{CH}_{3}-6\right) ;{ }^{13} \mathrm{C}-\mathrm{NMR}$ (DMSO-d, $150 \mathrm{MHz}$, ठ/ppm): $\delta 165.15$ (C-1'), 163.70 (C-5"), 163.32 (C-2"), 157.65 (C-2), 146.81 (C-4), 137.81 (C-3"'
\& C-5"'), 133.28 (C-4"'), 131.14 (C-2"' \& C-6"'), 122.07 (C-1"'), 108.09 (C-5), 35.38 (C-2'), 16.80 (C-6); El-MS: $m / z 366$ [M] $^{+}, 226\left[\mathrm{C}_{9} \mathrm{H}_{6} \mathrm{CIN}_{2} \mathrm{OS}\right]^{+}$, $179 \quad\left[\mathrm{C}_{8} \mathrm{H}_{4} \mathrm{CIN}_{2} \mathrm{OS}\right]^{+}, 139 \quad\left[\mathrm{C}_{5} \mathrm{H}_{3} \mathrm{~N}_{2} \mathrm{OS}\right]^{+}, 125$ $\left[\mathrm{C}_{7} \mathrm{H}_{5} \mathrm{Cl}\right]^{+}, 114\left[\mathrm{C}_{3} \mathrm{H}_{2} \mathrm{~N}_{2} \mathrm{OS}\right]^{+}, 75\left[\mathrm{C}_{6} \mathrm{H}_{3}\right]^{+}$.

\section{2-\{[5-(2,4-Dichlorophenyl)-1,3,4-oxadiazol-2- yl]sulfanyl\}-N-(4-methyl-1,3-thiazol-2- yl)acetamide (8h)}

Light-brown solid; yield: $77 \%$; m.p.: $168-169{ }^{\circ} \mathrm{C}$; Mol. Formula: $\mathrm{C}_{14} \mathrm{H}_{10} \mathrm{Cl}_{2} \mathrm{~N}_{4} \mathrm{O}_{2} \mathrm{~S}_{2}$; Mol. Mass: 400 $\mathrm{g} / \mathrm{mol}$; IR $\left(\mathrm{KBr}, \mathrm{cm}^{-1}\right) v_{\text {max }}: 3379(\mathrm{~N}-\mathrm{H}$ stretching), 2982 (C-H stretching of aromatic ring), 1677 ( $\mathrm{C}=\mathrm{N}$ stretching), 1647 ( $\mathrm{C}=\mathrm{O}$ stretching), 1575 $(\mathrm{C}=\mathrm{C}$ stretching of aromatic ring), 1164 (C-O-C stretching), 694 (C-Cl stretching); ${ }^{1} \mathrm{H}-\mathrm{NMR}$ $\left(\mathrm{DMSO}_{-} \mathrm{d}_{6}, 600 \mathrm{MHz}, \delta / \mathrm{ppm}\right): \delta 12.47$ (s, $1 \mathrm{H}$, CON-H), 7.96 (br.d, $J=8.5 \mathrm{~Hz}, 1 \mathrm{H}, \mathrm{H}-6$ "'), 7.90 (d, $\left.J=1.8 \mathrm{~Hz}, 1 \mathrm{H}, \mathrm{H}-3^{\prime \prime \prime}\right), 7.64$ (dd, $J=1.6,8.4$ Hz, 1H, H-5"'), 6.80 (br.s, 1H, H-5), 4.41 (br.s, $\left.2 \mathrm{H}, \quad \mathrm{CH}_{2}-2^{\prime}\right), 2.27$ (s, 3H, $\left.\mathrm{CH}_{3}-6\right) ;{ }^{13} \mathrm{C}-\mathrm{NMR}$ $\left(\mathrm{DMSO}-\mathrm{d}_{6}, 150 \mathrm{MHz}, \delta / \mathrm{ppm}\right): \delta 165.01$ (C-1'), 163.88 (C-5"), 162.65 (C-2"), 156.89 (C-2), 146.81 (C-4), 137.19 (C-4"'), 132.73 (C-2"'), 132.23 (C-6"'), 130.72 (C-3"'), 128.13 (C-5"'), 121.06 (C-1"'), 108.09 (C-5), 35.43 (C-2'), 16.80 (C-6); El-MS: $m / z 404[\mathrm{M}+4]^{+}, 402[\mathrm{M}+2]^{+}, \mathrm{m} / \mathrm{z}$ $400[\mathrm{M}]^{+}, 260\left[\mathrm{C}_{9} \mathrm{H}_{5} \mathrm{CIN}_{2} \mathrm{O}_{2} \mathrm{~S}\right]^{+}, 172\left[\mathrm{C}_{7} \mathrm{H}_{3} \mathrm{Cl}_{2} \mathrm{~N}\right]^{+}$, $141\left[\mathrm{C}_{5} \mathrm{H}_{5} \mathrm{~N}_{2} \mathrm{OS}\right]^{+}, 114 \quad\left[\mathrm{C}_{3} \mathrm{H}_{2} \mathrm{~N}_{2} \mathrm{OS}\right]^{+}, 100$ $\left[\mathrm{C}_{2} \mathrm{~N}_{2} \mathrm{OS}\right]^{+}$.

\section{2-\{[5-(3-Aminophenyl)-1,3,4-oxadiazol-2- yl]sulfanyl\}-N-(4-methyl-1,3-thiazol-2- yl)acetamide (8i)}

White amorphous solid; yield: 83\%; m.p.: 190$191{ }^{\circ} \mathrm{C}$; Mol. Formula: $\mathrm{C}_{14} \mathrm{H}_{13} \mathrm{~N}_{5} \mathrm{O}_{2} \mathrm{~S}_{2}$; Mol. Mass: $347 \mathrm{~g} / \mathrm{mol}$; IR $\left(\mathrm{KBr}, \mathrm{cm}^{-1}\right) \quad u_{\max }: 3378(\mathrm{~N}-\mathrm{H}$ stretching), 2962 (C-H stretching of aromatic ring), $1680 \quad(\mathrm{C}=\mathrm{N}$ stretching), $1653 \quad(\mathrm{C}=\mathrm{O}$ stretching), $1566(\mathrm{C}=\mathrm{C}$ stretching of aromatic ring), 1164 (C-O-C stretching); ${ }^{1} \mathrm{H}-\mathrm{NMR}$ (DMSO$\left.\mathrm{d}_{6}, 600 \mathrm{MHz}, \delta / \mathrm{ppm}\right): \delta 12.43(\mathrm{~s}, 1 \mathrm{H}, \mathrm{CON}-\mathrm{H})$, 7.93 (dd, $J=2.1,8.5 \mathrm{~Hz}, 1 \mathrm{H}, \mathrm{H}-6$ '"'), 7.88 (br.t, $J$ $\left.=8.5 \mathrm{~Hz}, 1 \mathrm{H}, \mathrm{H}-5^{\prime \prime \prime}\right), 7.48(\mathrm{~d}, J=2.1 \mathrm{~Hz}, 1 \mathrm{H}, \mathrm{H}-$ 2"'), 7.47 (dd, J = 2.1, $8.5 \mathrm{~Hz}, 1 \mathrm{H}, \mathrm{H}-4$ "'), 6.79 (s, $1 \mathrm{H}, \mathrm{H}-5$ ), 4.30 (br.s, 2H, $\mathrm{CH}_{2}-2$ ), 2.27 (s, 3H, $\left.\mathrm{CH}_{3}-6\right) ;{ }^{13} \mathrm{C}-\mathrm{NMR}$ (DMSO-d $\left.6,150 \mathrm{MHz}, \delta / \mathrm{ppm}\right): \delta$ 165.26 (C-1'), 162.99 (C-5"), 160.46 (C-2"), 157.71 (C-2), 146.80 (C-4), 132.21 (C-3"'), 132.04 (C-1"'), 129.41 (C-5"'), 129.38 (C-6"'), 126.35 (C-4'"), 126.02 (C-2'"), 108.05 (C-5), 35.36 (C-2'), 16.84 (C-6); El-MS: $m / z 347$ [M] $^{+}$, $207 \quad\left[\mathrm{C}_{9} \mathrm{H}_{8} \mathrm{~N}_{3} \mathrm{OS}\right]^{+}, \quad 193 \quad\left[\mathrm{C}_{8} \mathrm{H}_{6} \mathrm{~N}_{3} \mathrm{OS}\right]^{+}, 141$ $\left[\mathrm{C}_{5} \mathrm{H}_{5} \mathrm{~N}_{2} \mathrm{OS}\right]^{+}, 133\left[\mathrm{C}_{8} \mathrm{H}_{7} \mathrm{NO}^{+}, 118\left[\mathrm{C}_{3} \mathrm{HNOS}\right]^{+}, 92\right.$ $\left[\mathrm{C}_{6} \mathrm{H}_{6} \mathrm{~N}\right]^{+}, 65\left[\mathrm{C}_{5} \mathrm{H}_{5}\right]^{+}$. 
2-\{[5-(4-Aminophenyl)-1,3,4-oxadiazol-2yl]sulfanyl\}-N-(4-methyl-1,3-thiazol-2-yl) acetamide (8j)

White amorphous solid; yield: $82 \%$; m.p.: 215$216{ }^{\circ} \mathrm{C}$; Mol. Formula: $\mathrm{C}_{14} \mathrm{H}_{13} \mathrm{~N}_{5} \mathrm{O}_{2} \mathrm{~S}_{2}$; Mol. Mass: $347 \mathrm{~g} / \mathrm{mol}$; IR $\left(\mathrm{KBr}, \mathrm{cm}^{-1}\right) u_{\max }: 3376(\mathrm{~N}-\mathrm{H}$ stretching), 2966 (C-H stretching of aromatic ring), 1683 ( $C=N$ stretching), $1657 \quad(C=O$ stretching), $1563(C=C$ stretching of aromatic ring), 1168 (C-O-C stretching); ${ }^{1} \mathrm{H}-\mathrm{NMR}$ (DMSO$\left.\mathrm{d}_{6}, 600 \mathrm{MHz}, \delta / \mathrm{ppm}\right): \delta 12.43$ (s, 1H, CON-H), 7.56 (br.d, $J=8.5 \mathrm{~Hz}, 2 \mathrm{H}, \mathrm{H}-2$ '" \& H-6"'), 6.79 (s, $1 \mathrm{H}, \mathrm{H}-5$ ), 6.63 (br.d, $J=8.6 \mathrm{~Hz}, 2 \mathrm{H}, \mathrm{H}-3$ '" \& H$\left.5^{\prime \prime \prime}\right), 4.30$ (br.s, $2 \mathrm{H}, \mathrm{CH}_{2}-2$ '), 2.27 (s, 3H, $\mathrm{CH}_{3}-6$ ); ${ }^{13} \mathrm{C}-\mathrm{NMR}$ (DMSO-d $, 150 \mathrm{MHz}, \delta / \mathrm{ppm}$ ): $\delta 166.11$ (C-1'), 165.39 (C-5"), 160.54 (C-2"), 156.88 (C2), 152.40 (C-4"'), 146.90 (C-4), 127.90 (C-2'" \& C-6"'), 113.46 (C-3"' \& C-5"'), 109.13 (C-1"'), 108.00 (C-5), 35.31 (C-2'), 16.84 (C-6); El-MS: $\mathrm{m} / \mathrm{z} 347[\mathrm{M}]^{+}, 207\left[\mathrm{C}_{9} \mathrm{H}_{8} \mathrm{~N}_{3} \mathrm{OS}\right], 193\left[\mathrm{C}_{8} \mathrm{H}_{6} \mathrm{~N}_{3} \mathrm{OS}\right]$, $160\left[\mathrm{C}_{8} \mathrm{H}_{6} \mathrm{~N}_{3} \mathrm{O}\right]^{+}, 141\left[\mathrm{C}_{5} \mathrm{H}_{5} \mathrm{~N}_{2} \mathrm{OS}\right], 133\left[\mathrm{C}_{8} \mathrm{H}_{7} \mathrm{NO}\right]$, $118\left[\mathrm{C}_{3} \mathrm{HNOS}\right], 92\left[\mathrm{C}_{6} \mathrm{H}_{6} \mathrm{~N}\right], 65\left[\mathrm{C}_{5} \mathrm{H}_{5}\right]^{+}$.

\section{N-(4-Methyl-1,3-thiazol-2-yl)-2-\{[5-(2- nitrophenyl)-1,3,4-oxadiazol-2- yl]sulfanyl\}acetamide(8k)}

Light-yellow solid; yield: $77 \%$; m.p.: $170-171^{\circ} \mathrm{C}$; Mol. Formula: $\mathrm{C}_{14} \mathrm{H}_{11} \mathrm{~N}_{5} \mathrm{O}_{4} \mathrm{~S}_{2}$; Mol. Mass: 377 $\mathrm{g} / \mathrm{mol}$; IR $\left(\mathrm{KBr}, \mathrm{cm}^{-1}\right) v_{\text {max }}$ : 3364 (N-H stretching), 2982 (C-H stretching of aromatic ring), 1673 ( $\mathrm{C}=\mathrm{N}$ stretching), 1649 ( $\mathrm{C}=\mathrm{O}$ stretching), 1578 $(\mathrm{C}=\mathrm{C}$ stretching of aromatic ring), 1157 (C-O-C stretching); ${ }^{1} \mathrm{H}-\mathrm{NMR}$ (DMSO-d $6,600 \mathrm{MHz}$, $\delta / \mathrm{ppm}): \delta 12.47(\mathrm{~s}, 1 \mathrm{H}, \mathrm{CON}-\mathrm{H}), 8.19-8.17(\mathrm{~m}$, $\left.1 \mathrm{H}, \mathrm{H}-33^{\prime \prime}\right), 8.01-7.97(\mathrm{~m}, 1 \mathrm{H}, \mathrm{H}-6 " '), 7.94-7.90$ (m, 2H, H-4"' \& H-5"'), 6.80 (br.s, $1 \mathrm{H}, \mathrm{H}-5$ ), 4.41 (br.s, $2 \mathrm{H}, \mathrm{CH}_{2}-2^{\prime}$ ), 2.27 (s, 3H, $\mathrm{CH}_{3}-6$ ); ${ }^{13} \mathrm{C}-\mathrm{NMR}$ (DMSO-d $6,150 \mathrm{MHz}, \delta / \mathrm{ppm}$ ): $\delta 164.91$ (C-1'), 164.41 (C-5"), 161.69 (C-2"), 156.70 (C-2), 147.66 (C-2"'), 146.84 (C-4), 133.67 (C-4"'), 133.50 (C-5"'), 131.50 (C-3"'), 124.77 (C-6"'), 116.49 (C-1"'), 108.09 (C-5), 35.48 (C-2'), 16.81 (C-6); El-MS: m/z $377[\mathrm{MM}]^{+}, 227\left[\mathrm{C}_{8} \mathrm{H}_{7} \mathrm{~N}_{2} \mathrm{O}_{2} \mathrm{~S}_{2}\right]^{+}$, $141\left[\mathrm{C}_{5} \mathrm{H}_{5} \mathrm{~N}_{2} \mathrm{OS}\right]^{+}, 114\left[\mathrm{C}_{3} \mathrm{H}_{2} \mathrm{~N}_{2} \mathrm{OS}\right]^{+}, 76\left[\mathrm{C}_{6} \mathrm{H}_{4}\right]^{+}$.

\section{N-(4-Methyl-1,3-thiazol-2-yl)-2-\{[5-(3- nitrophenyl)-1,3,4-oxadiazol-2- yl]sulfanyl\}acetamide (8I)}

Light-yellow solid; yield: $78 \%$; m.p.: $194-195{ }^{\circ} \mathrm{C}$; Mol. Formula: $\mathrm{C}_{14} \mathrm{H}_{11} \mathrm{~N}_{5} \mathrm{O}_{4} \mathrm{~S}_{2}$; Mol. Mass: 377 $\mathrm{g} / \mathrm{mol}$; IR $\left(\mathrm{KBr}, \mathrm{cm}^{-1}\right) v_{\text {max }}: 3360$ (N-H stretching), 2963 (C-H stretching of aromatic ring), 1660 $(\mathrm{C}=\mathrm{N}$ stretching), $1650(\mathrm{C}=\mathrm{O}$ stretching $), 1562$ $(\mathrm{C}=\mathrm{C}$ stretching of aromatic ring), 1169 (C-O-C stretching); ${ }^{1} \mathrm{H}-\mathrm{NMR}$ (DMSO-d $6,600 \mathrm{MHz}$, $\delta / \mathrm{ppm}): \delta 12.48$ (s, $1 \mathrm{H}, \mathrm{CON}-\mathrm{H}), 8.63$ (br.s, $J=$ $\left.7.7 \mathrm{~Hz}, 1 \mathrm{H}, \mathrm{H}-2{ }^{\prime \prime \prime}\right), 8.46$ (dd, $J=1.8,8.2 \mathrm{~Hz}, 1 \mathrm{H}$,
H-4"'), 8.37 (br.d, $J=7.8 \mathrm{~Hz}, 1 \mathrm{H}, \mathrm{H}-6$ '"') $^{\prime} 7.88$ (br.t, 1H, H-5'"), 6.79 (br.s, 1H, H-5), 4.40 (br.s, $\left.2 \mathrm{H}, \quad \mathrm{CH}_{2}-2^{\prime}\right), 2.26\left(\mathrm{~s}, 3 \mathrm{H}, \quad \mathrm{CH}_{3}-6\right) ;{ }^{13} \mathrm{C}-\mathrm{NMR}$ (DMSO-d $6,150 \mathrm{MHz}, \delta / \mathrm{ppm}): \delta 165.12$ (C-1'), 163.97 (C-5"), 163.70 (C-2"), 157.63 (C-2), 148.17 (C-3"'), 148.15 (C-4), 126.30 (C-4"'), 131.23 (C-5"'), 132.34 (C-6"'), 124.33 (C-1"'), 120.82 (C-2'"'), 108.91 (C-5), 35.36 (C-2'), 16.73 (C-6); El-MS: $m / z 377[\mathrm{M}]^{+}, 227\left[\mathrm{C}_{8} \mathrm{H}_{7} \mathrm{~N}_{2} \mathrm{O}_{2} \mathrm{~S}_{2}\right]^{+}$, $150\left[\mathrm{C}_{7} \mathrm{H}_{4} \mathrm{NO}_{3}\right]^{+}, \quad 141 \quad\left[\mathrm{C}_{5} \mathrm{H}_{5} \mathrm{~N}_{2} \mathrm{OS}\right]^{+}, \quad 114$ $\left[\mathrm{C}_{3} \mathrm{H}_{2} \mathrm{~N}_{2} \mathrm{OS}\right]^{+}, 76\left[\mathrm{C}_{6} \mathrm{H}_{4}\right]^{+}$.

\section{N-(4-Methyl-1,3-thiazol-2-yl)-2-\{[5-(4- nitrophenyl)-1,3,4-oxadiazol-2- yl]sulfanyl\}acetamide (8m)}

Light-yellow solid; yield: $77 \%$; m.p.: $173-174{ }^{\circ} \mathrm{C}$; Mol. Formula: $\mathrm{C}_{14} \mathrm{H}_{11} \mathrm{~N}_{5} \mathrm{O}_{4} \mathrm{~S}_{2}$; Mol. Mass: 377 $\mathrm{g} / \mathrm{mol}$; IR $\left(\mathrm{KBr}, \mathrm{cm}^{-1}\right) v_{\text {max }}: 3364$ (N-H stretching), 2987 (C-H stretching of aromatic ring), 1684 ( $\mathrm{C}=\mathrm{N}$ stretching), 1654 ( $\mathrm{C}=\mathrm{O}$ stretching), 1586 ( $\mathrm{C}=\mathrm{C}$ stretching of aromatic ring), 1173 (C-O-C stretching); ${ }^{1} \mathrm{H}-\mathrm{NMR}$ (DMSO-d $6,600 \mathrm{MHz}$, $\delta / \mathrm{ppm}$ ): $\delta 12.48$ (s, $1 \mathrm{H}, \mathrm{CON}-\mathrm{H}), 8.42$ (br.d, $J=$ $7.0 \mathrm{~Hz}, 1 \mathrm{H}, \mathrm{H}-3$ "' \& H-5"'), 8.21 (br.d, $J=7.0 \mathrm{~Hz}$, $2 \mathrm{H}, \mathrm{H}-2$ '" \& H-6"'), 6.79 (br.s, $1 \mathrm{H}, \mathrm{H}-5$ ), 4.40 (br.s, $\left.2 \mathrm{H}, \mathrm{CH}_{2}-2^{\prime}\right), 2.26$ (s, 3H, $\mathrm{CH}_{3}-6$ ); ${ }^{13} \mathrm{C}-\mathrm{NMR}$ (DMSO-d $\left.\mathrm{d}_{6}, 150 \mathrm{MHz}, \delta / \mathrm{ppm}\right): \delta 165.00$ (C-1'), 164.51 (C-5"), 163.93 (C-2"), 156.01 (C-2), 149.15 (C-4"'), 148.15 (C-4), 127.74 (C-2"' \& C6"'), 124.58 (C-3"' \& C-5"'), 122.01 (C-1"'), 108.12 (C-5), 35.38 (C-2'), 16.73 (C-6); El-MS:

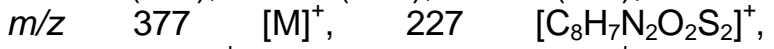
$\begin{array}{llll}150\left[\mathrm{C}_{7} \mathrm{H}_{4} \mathrm{NO}_{3}\right]^{+}, & 141 & {\left[\mathrm{C}_{5} \mathrm{H}_{5} \mathrm{~N}_{2} \mathrm{OS}\right]^{+},} & 114\end{array}$ $\left[\mathrm{C}_{3} \mathrm{H}_{2} \mathrm{~N}_{2} \mathrm{OS}\right]^{+}, 76\left[\mathrm{C}_{6} \mathrm{H}_{4}\right]^{+}$.

\section{2-\{[5-(3,5-Dinitrophenyl)-1,3,4-oxadiazol-2- yl]sulfanyl\}-N-(4-methyl-1,3-thiazol-2- yl)acetamide(8n)}

Light-green solid; yield: $74 \%$; m.p.: $215-216{ }^{\circ} \mathrm{C}$; Mol. Formula: $\mathrm{C}_{14} \mathrm{H}_{10} \mathrm{~N}_{6} \mathrm{O}_{6} \mathrm{~S}_{2}$; Mol. Mass: 422 $\mathrm{g} / \mathrm{mol}$; IR $\left(\mathrm{KBr}, \mathrm{cm}^{-1}\right) v_{\max }: 3366$ (N-H stretching), 2985 (C-H stretching of aromatic ring), 1685 ( $\mathrm{C}=\mathrm{N}$ stretching), 1658 ( $\mathrm{C}=\mathrm{O}$ stretching), 1589 ( $\mathrm{C}=\mathrm{C}$ stretching of aromatic ring), 1192 (C-O-C stretching); ${ }^{1} \mathrm{H}-\mathrm{NMR} \quad$ (DMSO-d $6,600 \mathrm{MHz}$, $\delta / \mathrm{ppm}$ ): $\delta 12.48$ (s, $1 \mathrm{H}, \mathrm{CON}-\mathrm{H}), 7.98$ (br.d, $J=$ $1.6 \mathrm{~Hz}, 2 \mathrm{H}, \mathrm{H}-2$ "' \& H-6"'), 7.66 (br.s, $1 \mathrm{H}, \mathrm{H}-4$ "'), 6.79 (br.s, $1 \mathrm{H}, \mathrm{H}-5$ ), 4.40 (br.s, $2 \mathrm{H}, \mathrm{CH}_{2}-2$ '), 2.26 (s, 3H, $\left.\mathrm{CH}_{3}-6\right) ;{ }^{13} \mathrm{C}-\mathrm{NMR}$ (DMSO-d, $150 \mathrm{MHz}$, $\delta / \mathrm{ppm}): \delta 165.12$ (C-1'), 163.88 (C-5"), 162.67 (C-2"), 157.71 (C-2), 145.23 (C-4), 132.60 (C1"'), 132.24 (C-2"' \& C-6"'), 131.97 (C-3"' \& C5"'), 128.19 (C-4"'), 108.91 (C-5), 35.40 (C-2'), $16.70 \quad$ (C-6); EI-MS: $\mathrm{m} / \mathrm{z} \quad 422 \quad[\mathrm{M}]^{+}, \quad 303$ $\left[\mathrm{C}_{12} \mathrm{H}_{8} \mathrm{~N}_{4} \mathrm{O}_{2} \mathrm{~S}_{2}\right]^{+}, \quad 193 \quad\left[\mathrm{C}_{8} \mathrm{H}_{6} \mathrm{~N}_{3} \mathrm{OS}\right]^{+}, \quad 140$ $\left[\mathrm{C}_{5} \mathrm{H}_{5} \mathrm{~N}_{2} \mathrm{OS}\right]^{+}, 114\left[\mathrm{C}_{3} \mathrm{H}_{2} \mathrm{~N}_{2} \mathrm{OS}\right]^{+}, 104\left[\mathrm{C}_{7} \mathrm{H}_{4} \mathrm{O}\right]^{+}, 76$ $\left[\mathrm{C}_{6} \mathrm{H}_{4}\right]^{+}$. 


\section{2-\{[5-(2-Methyl-3,5-dinitrophenyl)-1,3,4- oxadiazol-2-yl]sulfanyl\}-N-(4-methyl-1,3- thiazol-2-yl)acetamide (80)}

Light-brown solid; yield: $71 \%$; m.p.: $191-192{ }^{\circ} \mathrm{C}$; Mol. Formula: $\mathrm{C}_{15} \mathrm{H}_{12} \mathrm{~N}_{6} \mathrm{O}_{6} \mathrm{~S}_{2}$; Mol. Mass: 436 $\mathrm{g} / \mathrm{mol}$; IR $\left(\mathrm{KBr}, \mathrm{cm}^{-1}\right) v_{\text {max }}: 3346$ (N-H stretching), 2969 (C-H stretching of aromatic ring), 1665 $(\mathrm{C}=\mathrm{N}$ stretching), $1636 \quad(\mathrm{C}=\mathrm{O}$ stretching), 1568 $(\mathrm{C}=\mathrm{C}$ stretching of aromatic ring), 1152 (C-O-C stretching); ${ }^{1} \mathrm{H}-\mathrm{NMR} \quad$ (DMSO-d $6,600 \mathrm{MHz}$, $\delta / \mathrm{ppm}): \delta 12.40(\mathrm{~s}, 1 \mathrm{H}, \mathrm{CON}-\mathrm{H}), 7.90(\mathrm{~d}, J=2.8$ $\left.\mathrm{Hz}, 1 \mathrm{H}, \mathrm{H}-4{ }^{\prime \prime \prime}\right), 7.64$ (d, J = $\left.2.8 \mathrm{~Hz}, 1 \mathrm{H}, \mathrm{H}-6^{\prime \prime \prime}\right)$, 6.79 (br.s, 1H, H-5), 4.24 (br.s, $2 \mathrm{H}, \mathrm{CH}_{2}-2$ '), 2.34 (br.s, 3H, $\left.\mathrm{CH}_{3}-\mathrm{C}^{\prime \prime \prime}\right), 2.27$ (s, 3H, $\mathrm{CH}_{3}-6$ ); ${ }^{13} \mathrm{C}-\mathrm{NMR}$ (DMSO-d $6,150 \mathrm{MHz}, \delta / \mathrm{ppm}$ ): $\delta 165.12$ (C-1'), 163.88 (C-5"), 162.67 (C-2"), 157.71 (C-2), 146.88 (C-4), 132.60 (C-1"'), 132.24 (C-6"'), 131.97 (C-5"'), 130.81 (C-3"'), 128.19 (C-4"'), 128.14 (C-2"'), 108.07 (C-5), 35.40 (C-2'), 20.67 (C-7"'), 16.82 (C-6"'); El-MS: m/z 436 [M] ${ }^{+}, 303$ $\left[\mathrm{C}_{12} \mathrm{H}_{8} \mathrm{~N}_{4} \mathrm{O}_{2} \mathrm{~S}_{2}\right]^{+}, \quad 281 \quad\left[\mathrm{C}_{9} \mathrm{H}_{5} \mathrm{~N}_{4} \mathrm{O}_{5} \mathrm{~S}\right]^{+}, \quad 193$ $\left[\mathrm{C}_{8} \mathrm{H}_{6} \mathrm{~N}_{3} \mathrm{OS}\right]^{+}, \quad 181 \quad\left[\mathrm{C}_{7} \mathrm{H}_{5} \mathrm{~N}_{2} \mathrm{O}_{4}\right]^{+}, \quad 140$ $\left[\mathrm{C}_{5} \mathrm{H}_{5} \mathrm{~N}_{2} \mathrm{OS}\right]^{+}, 114\left[\mathrm{C}_{3} \mathrm{H}_{2} \mathrm{~N}_{2} \mathrm{OS}\right]^{+}, 104\left[\mathrm{C}_{7} \mathrm{H}_{4} \mathrm{O}\right]^{+}$.

\section{2-\{[5-(2-Chloro-3,5-dinitrophenyl)-1,3,4- oxadiazol-2-yl]sulfanyl\}-N-(4-methyl-1,3- thiazol-2-yl)acetamide (8p)}

Light-brown solid; yield: $77 \%$; m.p.: $177-178{ }^{\circ} \mathrm{C}$; Mol. Formula: $\mathrm{C}_{14} \mathrm{H}_{9} \mathrm{CIN}_{6} \mathrm{O}_{6} \mathrm{~S}_{2}$; Mol. Mass: 456 $\mathrm{g} / \mathrm{mol}$; IR (KBr, $\left.\mathrm{cm}^{-1}\right) v_{\text {max }}: 3354$ (N-H stretching), 2978 (C-H stretching of aromatic ring), 1674 ( $\mathrm{C}=\mathrm{N}$ stretching), 1645 ( $\mathrm{C}=\mathrm{O}$ stretching), 1576 $(\mathrm{C}=\mathrm{C}$ stretching of aromatic ring), 1163 (C-O-C stretching), 693 (C-Cl stretching); ${ }^{1} \mathrm{H}-\mathrm{NMR}$ $\left(\right.$ DMSO-d $_{6}, 600 \mathrm{MHz}, \delta / \mathrm{ppm}$ ): $\delta 12.40$ (s, $1 \mathrm{H}$, CON-H), 7.91 (d, $J=2.6 \mathrm{~Hz}, 1 \mathrm{H}, \mathrm{H}-4$ "'), 7.68 (d, $\left.J=2.6 \mathrm{~Hz}, 1 \mathrm{H}, \mathrm{H}-6{ }^{\prime \prime \prime}\right), 6.79$ (br.s, $\left.1 \mathrm{H}, \mathrm{H}-5\right), 4.24$ (br.s, 2H, $\mathrm{CH}_{2}-2^{\prime}$ ), 2.27 (s, 3H, $\mathrm{CH}_{3}-6$ ); ${ }^{13} \mathrm{C}-\mathrm{NMR}$ (DMSO-d $6,150 \mathrm{MHz}, \delta / \mathrm{ppm}$ ): $\delta 165.12$ (C-1'), 163.88 (C-5"), 162.67 (C-2"), 157.71 (C-2), 147.88 (C-4), 132.60 (C-1"'), 132.24 (C-6"'), 131.97 (C-5"'), 130.81 (C-3"'), 128.19 (C-4"'), 128.14 (C-2"'), 108.07 (C-5), 35.40 (C-2'), 16.82 (C-6); El-MS: $m / z 456[\mathrm{M}]^{+}, 303\left[\mathrm{C}_{12} \mathrm{H}_{8} \mathrm{~N}_{4} \mathrm{O}_{2} \mathrm{~S}_{2}\right]^{+}$, $302\left[\mathrm{C}_{8} \mathrm{H}_{2} \mathrm{CIN}_{4} \mathrm{O}_{5} \mathrm{~S}\right]^{+}, 227\left[\mathrm{C}_{9} \mathrm{H}_{6} \mathrm{CIN}_{2} \mathrm{OS}\right]^{+}, 201$ $\left[\mathrm{C}_{6} \mathrm{H}_{2} \mathrm{CIN}_{2} \mathrm{O}_{4}\right]^{+}, \quad 193 \quad\left[\mathrm{C}_{8} \mathrm{H}_{6} \mathrm{~N}_{3} \mathrm{OS}\right]^{+}, \quad 141$ $\left[\mathrm{C}_{5} \mathrm{H}_{5} \mathrm{~N}_{2} \mathrm{OS}\right]^{+}, 114\left[\mathrm{C}_{3} \mathrm{H}_{2} \mathrm{~N}_{2} \mathrm{OS}\right]^{+}, 104\left[\mathrm{C}_{7} \mathrm{H}_{4} \mathrm{O}\right]^{+}, 76$ $\left[\mathrm{C}_{6} \mathrm{H}_{4}\right]^{+}$.

\section{2-[(5-Benzyl-1,3,4-oxadiazol-2-yl)sulfanyl]-N- (4-methyl-1,3-thiazol-2-yl)acetamide(8q)}

Dull-white solid; yield: $80 \%$; m.p.: $178-179{ }^{\circ} \mathrm{C}$, Mol. Formula: $\mathrm{C}_{15} \mathrm{H}_{14} \mathrm{~N}_{4} \mathrm{O}_{2} \mathrm{~S}_{2}$; Mol. Mass: 346 $\mathrm{g} / \mathrm{mol}$; IR $\left(\mathrm{KBr}, \mathrm{cm}^{-1}\right) v_{\text {max }}: 3357$ (N-H stretching), 2974 (C-H stretching of aromatic ring), 1682 ( $\mathrm{C}=\mathrm{N}$ stretching), $1646 \quad(\mathrm{C}=\mathrm{O}$ stretching), 1582 $(\mathrm{C}=\mathrm{C}$ stretching of aromatic ring), 1166 (C-O-C stretching); ${ }^{1} \mathrm{H}-\mathrm{NMR} \quad$ (DMSO-d $6,600 \mathrm{MHz}$, $\delta / \mathrm{ppm}): \delta 12.40$ (s, $1 \mathrm{H}, \mathrm{CON}-\mathrm{H}), 7.32-7.25(\mathrm{~m}$, $5 \mathrm{H}, \mathrm{H}-2$ "' to $\mathrm{H}-6$ '"'), 6.79 (br.s, $1 \mathrm{H}, \mathrm{H}-5$ ), 4.33 (br.s, 2H, $\mathrm{CH}_{2}-7$ '"') $^{2}, 4.24$ (br.s, $2 \mathrm{H}, \mathrm{CH}_{2}-2$ '), 2.27 (s, 3H, $\mathrm{CH}_{3}-6$ ); ${ }^{13} \mathrm{C}-\mathrm{NMR}$ (DMSO-d, $150 \mathrm{MHz}$, $\delta / \mathrm{ppm}): \delta 166.73$ (C-1'), 165.01 (C-5"), 162.38 (C-2"), 157.01 (C-2), 147.01 (C-4), 134.13 (C1"'), 128.79 (C-3"' \& C-5"'), 128.69 (C-2"' \& C6"'), 127.22 (C-4"'), 108.07 (C-5), 35.27 (C-2'), 30.67 (C-7"'), 16.82 (C-6); El-MS: $m / z 346$ [M] ${ }^{+}$, $255\left[\mathrm{C}_{8} \mathrm{H}_{7} \mathrm{~N}_{4} \mathrm{O}_{2} \mathrm{~S}\right]^{+}, 228 \quad\left[\mathrm{C}_{7} \mathrm{H}_{7} \mathrm{~N}_{4} \mathrm{OS}_{2}\right]^{+}, \quad 206$ $\left[\mathrm{C}_{10} \mathrm{H}_{9} \mathrm{~N}_{2} \mathrm{OS}\right]^{+}, \quad 192 \quad\left[\mathrm{C}_{8} \mathrm{H}_{5} \mathrm{~N}_{3} \mathrm{OS}\right]^{+}, \quad 141$ $\left[\mathrm{C}_{5} \mathrm{H}_{5} \mathrm{~N}_{2} \mathrm{OS}\right]^{+}, 114\left[\mathrm{C}_{3} \mathrm{H}_{2} \mathrm{~N}_{2} \mathrm{OS}\right]^{+}, 91\left[\mathrm{C}_{7} \mathrm{H}_{7}\right]^{+}, 83$ $\left[\mathrm{C}_{3} \mathrm{H}_{3} \mathrm{~N}_{2} \mathrm{O}\right]^{+}$.

\section{N-(4-Methyl-1,3-thiazol-2-yl)-2-\{[5- (naphthalen-1-ylmethyl)-1,3,4-oxadiazol-2- yl]sulfanyl\}acetamide(8r)}

Light-yellow solid; yield: $78 \%$; m.p.: $206-207{ }^{\circ} \mathrm{C}$; Mol. Formula: $\mathrm{C}_{18} \mathrm{H}_{14} \mathrm{~N}_{4} \mathrm{O}_{2} \mathrm{~S}_{2}$; Mol. Mass: 382 $\mathrm{g} / \mathrm{mol}$; IR $\left(\mathrm{KBr}, \mathrm{cm}^{-1}\right) v_{\max }: 3359$ (N-H stretching), 2978 (C-H stretching of aromatic ring), 1677 ( $\mathrm{C}=\mathrm{N}$ stretching), $1646 \quad(\mathrm{C}=\mathrm{O}$ stretching), 1579 ( $\mathrm{C}=\mathrm{C}$ stretching of aromatic ring), 1163 (C-O-C stretching); ${ }^{1} \mathrm{H}-\mathrm{NMR}$ (DMSO-d $6,600 \mathrm{MHz}$, $\delta / \mathrm{ppm}): \delta 12.40$ (s, $1 \mathrm{H}, \mathrm{CON}-\mathrm{H}), 8.07$ (br.d, $J=$ $7.9 \mathrm{~Hz}, 1 \mathrm{H}, \mathrm{H}-6$ '"'), 7.95 (dist.dd, $J=1.5,7.4 \mathrm{~Hz}$, $1 \mathrm{H}, \mathrm{H}-5$ "'), 7.88 (dist.dd, $J=1.5,7.1 \mathrm{~Hz}, 1 \mathrm{H}, \mathrm{H}-$ 4"'), 7.57-7.53 (m, 2H, H-7"' \& H-8"'), 7.48-7.44 (m, 2H, H-2"'\& H-3"'), 6.79 (br.s, $1 \mathrm{H}, \mathrm{H}-5$ ), 4.59 (s, 2H, $\mathrm{CH}_{2}-11$ "'), 4.24 (br.s, $2 \mathrm{H}, \mathrm{CH}_{2}-2$ '), 2.27 (s, $\left.3 \mathrm{H}, \mathrm{CH}_{3}-6\right)$; ${ }^{13} \mathrm{C}-\mathrm{NMR}$ (DMSO-d 6 , $150 \mathrm{MHz}$, $\delta / \mathrm{ppm}): \delta 166.73$ (C-1'), 164.96 (C-5"), 164.76 (C-2"), 155.76 (C-2), 148.56 (C-1"'), 147.0 (C-4), 133.39 (C-7"'), 131.22 ( C-6"'), 130.22 (C-3"'), 128.56 (C-4"'), 128.11 (C-5"'), 127.63 (C-8"'), 126.50 (C-2"'), 125.98 (C-9"'), 123.62 (C-10"'), 108.07 (C-5), 35.24 (C-2'), 28.44 (C-11'"'), 16.82 (C-6); El-MS: $m / z 382[\mathrm{M}]^{+}, 255\left[\mathrm{C}_{8} \mathrm{H}_{7} \mathrm{~N}_{4} \mathrm{O}_{2} \mathrm{~S}\right]^{+}$, $228\left[\mathrm{C}_{7} \mathrm{H}_{7} \mathrm{~N}_{4} \mathrm{OS}_{2}\right]^{+}, \quad 242\left[\mathrm{C}_{13} \mathrm{H}_{10} \mathrm{~N}_{2} \mathrm{OS}\right]^{+}, \quad 241$ $\left[\begin{array}{llll}\left.\mathrm{C}_{13} \mathrm{H}_{9} \mathrm{~N}_{2} \mathrm{OS}\right]^{+}, & 141 \quad\left[\mathrm{C}_{5} \mathrm{H}_{5} \mathrm{~N}_{2} \mathrm{OS}\right]^{+}, & 114\end{array}\right.$ $\left[\mathrm{C}_{3} \mathrm{H}_{2} \mathrm{~N}_{2} \mathrm{OS}\right]^{+}, 141\left[\mathrm{C}_{11} \mathrm{H}_{9}\right]^{+}, 83\left[\mathrm{C}_{3} \mathrm{H}_{3} \mathrm{~N}_{2} \mathrm{O}\right]^{+}$.

\section{Enzyme inhibition}

In the search for new more effective therapeutic agents, the synthesized bi-heterocycles 8a-p were screened for their inhibitory activity against four pharmacologically relevant enzymes: acetylcholinesterase, butyrylcholinesterase, urease and $\alpha$-glucosidase. The results of enzyme inhibition are represented as \% inhibition and $\mathrm{IC}_{50}$ for cholinesterases, urease, glucosidase (Table 2) and $\mathrm{LD}_{50}$ values for brine shrimps (Table 3).

The structural verification was performed by spectral analysis. The structures of these derivatives were deduced based on IR, EI-MS, 
Table 2: Inhibition (\%) and $\mathrm{IC}_{50}$ values for acetylcholinesterase, butyrylcholinesterase, $\alpha$-glucosidase and urease.

\begin{tabular}{|c|c|c|c|c|c|c|c|c|}
\hline \multirow[t]{2}{*}{ Compd. } & \multicolumn{2}{|c|}{ AChE inhibition } & \multicolumn{2}{|c|}{ BChE inhibition } & \multicolumn{2}{|c|}{$\alpha-$ Glucosidase inhibition } & \multicolumn{2}{|c|}{ Urease inhibition } \\
\hline & $\begin{array}{c}\text { Inhibition (\%) } \\
\text { at } 0.5 \mathrm{mM}\end{array}$ & $I_{50}(\mu M)$ & $\begin{array}{c}\text { Inhibition (\%) } \\
\text { at } 0.5 \mathrm{mM}\end{array}$ & $I_{50}(\mu M)$ & $\begin{array}{c}\text { Inhibition (\%) } \\
\text { at } 0.5 \mathrm{mM}\end{array}$ & $I C_{50}(\mu M)$ & $\begin{array}{c}\text { Inhibition (\%) } \\
\text { at } 0.5 \mathrm{mM}\end{array}$ & $I_{50}(\mu M)$ \\
\hline $8 a$ & $36.12 \pm 0.19$ & - & $36.52 \pm 0.29$ & - & $56.21 \pm 0.30$ & $112.13 \pm 0.16$ & $46.52 \pm 0.19$ & - \\
\hline $8 b$ & $84.31 \pm 0.24$ & $43.85 \pm 0.12$ & $47.27 \pm 0.26$ & - & $67.14 \pm 0.19$ & $342.13 \pm 0.16$ & $69.31 \pm 0.31$ & $254.11 \pm 0.12$ \\
\hline $8 c$ & $89.31 \pm 0.35$ & $49.25 \pm 0.09$ & $58.14 \pm 0.25$ & $129.15 \pm 0.11$ & $51.32 \pm 0.14$ & $>500$ & $77.13 \pm 0.20$ & $291.47 \pm 0.19$ \\
\hline $8 d$ & $86.52 \pm 0.29$ & $39.47 \pm 0.13$ & $54.62 \pm 0.34$ & $438.52 \pm 0.22$ & $32.25 \pm 0.13$ & . & $56.95 \pm 0.37$ & $223.15 \pm 0.14$ \\
\hline $8 e$ & $86.28 \pm 0.36$ & $49.73 \pm 0.15$ & $12.23 \pm 0.35$ & - & $86.41 \pm 0.17$ & $192.27 \pm 0.14$ & $76.31 \pm 0.50$ & $250.30 \pm 0.25$ \\
\hline $8 f$ & $83.74 \pm 0.23$ & $53.15 \pm 0.25$ & $45.27 \pm 0.35$ & - & $79.11 \pm 0.21$ & $187.35 \pm 0.11$ & $92.35 \pm 0.31$ & $161.26 \pm 0.23$ \\
\hline $8 g$ & $77.31 \pm 0.15$ & $79.25 \pm 0.05$ & $45.14 \pm 0.35$ & - & $77.23 \pm 0.19$ & $201.23 \pm 0.21$ & $30.61 \pm 0.29$ & $228.13 \pm 0.14$ \\
\hline $8 h$ & $71.36 \pm 0.64$ & $119.53 \pm 0.21$ & $71.42 \pm 0.45$ & $238.64 \pm 0.18$ & $78.12 \pm 0.18$ & $107.42 \pm 0.12$ & $41.56 \pm 0.15$ & - \\
\hline $8 i$ & $92.53 \pm 0.45$ & $72.85 \pm 0.24$ & $52.74 \pm 0.63$ & $464.27 \pm 0.19$ & $87.15 \pm 0.19$ & $156.29 \pm 0.15$ & $67.28 \pm 0.41$ & $212.31 \pm 0.12$ \\
\hline $8 j$ & $87.85 \pm 0.42$ & $92.74 \pm 0.15$ & $79.26 \pm 0.15$ & $67.85 \pm 0.11$ & $82.11 \pm 0.18$ & $278.21 \pm 0.15$ & - & - \\
\hline $8 k$ & $87.61 \pm 0.27$ & $29.53 \pm 0.12$ & $82.47 \pm 0.16$ & $56.23 \pm 0.09$ & $31.13 \pm 0.15$ & - & - & - \\
\hline 81 & $91.74 \pm 0.13$ & $17.25 \pm 0.07$ & $15.47 \pm 0.27$ & - & $91.83 \pm 0.15$ & $62.23 \pm 0.13$ & $10.26 \pm 0.20$ & - \\
\hline $8 m$ & $90.71 \pm 0.14$ & $36.84 \pm 0.08$ & $67.35 \pm 0.36$ & $109.25 \pm 0.21$ & $91.48 \pm 0.15$ & $61.25 \pm 0.11$ & $49.24 \pm 0.51$ & - \\
\hline $8 n$ & $87.21 \pm 0.32$ & $63.80 \pm 0.45$ & $38.27 \pm 0.29$ & - & $77.51 \pm 0.55$ & $287.13 \pm 0.12$ & $47.28 \pm 0.15$ & - \\
\hline 80 & $90.12 \pm 0.29$ & $49.47 \pm 0.13$ & $58.62 \pm 0.34$ & $238.52 \pm 0.22$ & $91.59 \pm 0.23$ & $57.35 \pm 0.17$ & $60.54 \pm 0.17$ & $249.35 \pm 0.15$ \\
\hline $8 p$ & $66.42 \pm 0.17$ & $59.47 \pm 0.25$ & $78.62 \pm 0.34$ & $137.52 \pm 0.22$ & $63.48 \pm 0.17$ & $293.15 \pm 0.19$ & $35.67 \pm 0.10$ & - \\
\hline $8 q$ & $89.27 \pm 0.15$ & $18.36 \pm 0.08$ & $65.15 \pm 0.45$ & $292.14 \pm 0.16$ & $52.15 \pm 0.17$ & $>500$ & $61.45 \pm 0.40$ & $247.35 \pm 0.18$ \\
\hline $8 r$ & $37.50 \pm 0.23$ & - & . & - & $69.24 \pm 0.27$ & $77.35 \pm 0.11$ & $27.52 \pm 0.19$ & - \\
\hline Eserine & $91.27 \pm 1.17$ & $0.04 \pm 0.0001$ & $82.82 \pm 1.09$ & $0.85 \pm 0.0001$ & & & & \\
\hline Acarbose & & & & & $92.23 \pm 0.16$ & $37.38 \pm 0.12$ & & \\
\hline Thiourea & & & & & & & $98.12 \pm 0.18$ & $21.11 \pm 0.12$ \\
\hline
\end{tabular}

Note: All compounds were dissolved in methanol and experiments were performed in triplicate (mean \pm SEM, $\mathrm{n}=$ 3). AChE = acetylcholinesterase, BChE =butyrylcholinesterase.

Table 3: Brine shrimp activity

\begin{tabular}{lccccccc}
\hline Compd. & LD $_{50}(\mathbf{m M})$ & Compd. & LD $_{50}(\mathbf{m M})$ & Compd. & LD $_{50}(\mathbf{m M})$ & Compd. & LD $_{50}(\mathbf{m M})$ \\
\hline $\mathbf{8 a}$ & 45.21 & $\mathbf{8 f}$ & 243.6 & $\mathbf{8 k}$ & 358.4 & $\mathbf{8 p}$ & 201.1 \\
$\mathbf{8 b}$ & 213.5 & $\mathbf{8 g}$ & 354.4 & $\mathbf{8 l}$ & 466.7 & $\mathbf{8 q}$ & 198.5 \\
$\mathbf{8 c}$ & 122.7 & $\mathbf{8 h}$ & 395.4 & $\mathbf{8 m}$ & 77.34 & $\mathbf{8 r}$ & 61.47 \\
$\mathbf{8 d}$ & 498.7 & $\mathbf{8 i}$ & 459.7 & $\mathbf{8 n}$ & 171.8 & Doxo- & 5.21 \\
$\mathbf{8 e}$ & 521.1 & $\mathbf{8 j}$ & 365.8 & $\mathbf{8 0}$ & 189.7 & rubicin & \\
\hline
\end{tabular}

Note: Doxorubicin was used as standard

${ }^{1} \mathrm{H}-\mathrm{NMR}$ and ${ }^{13} \mathrm{C}-\mathrm{NMR}$ spectral data. The structural analysis of one of the compounds is discussed here in detail for the convenience of the readers. The molecule, $\mathbf{8 e}$, was obtained as a light-brown solid with $77 \%$ yield and had a melting point of $158-159{ }^{\circ} \mathrm{C}$. Its molecular formula, $\mathrm{C}_{14} \mathrm{H}_{11} \mathrm{CIN}_{4} \mathrm{O}_{2} \mathrm{~S}_{2}$, was established by identifying the $[\mathrm{M}]^{+}$ion peak at $\mathrm{m} / \mathrm{z} 366$, in the ElMS spectrum and counting the number of protons in the ${ }^{1} \mathrm{H}$-NMR spectrum. The number of carbons in the ${ }^{13} \mathrm{C}$-NMR spectrum also supported the assignment of its molecular formula. In the El-MS spectrum, the peak at $m / z 368$ was identified as the $[\mathrm{M}+2]^{+}$peak, while the molecular ion peak was observed at $\mathrm{m} / \mathrm{z} 366$ along with some peculiar fragment ion peaks as indicated in the spectral characterization section. The 2chlorophenyl moiety in the molecule was rationalized by four typical signals in the aromatic region of the ${ }^{1} \mathrm{H}$-NMR spectrum (Figure 2 ) at $\delta$ 7.94 (dd, $\left.J=1.6,7.8 \mathrm{~Hz}, 1 \mathrm{H}, \mathrm{H}-3{ }^{\prime \prime \prime}\right), 7.69$ (br.d, $J$ $\left.=7.9 \mathrm{~Hz}, 1 \mathrm{H}, \mathrm{H}-6^{\prime \prime \prime}\right), 7.62(\mathrm{dt}, J=1.6,8.1 \mathrm{~Hz}, 1 \mathrm{H}$, $\left.\mathrm{H}-4{ }^{\prime \prime \prime}\right)$, and 7.53 (dt, $\left.J=0.9,6.6 \mathrm{~Hz}, 1 \mathrm{H}, \mathrm{H}-5^{\prime \prime \prime}\right)$. Similarly, the 4-methyl-1,3-thiazole moiety was deduced through two peaks at $\delta 6.80$ (br.s, $1 \mathrm{H}$,

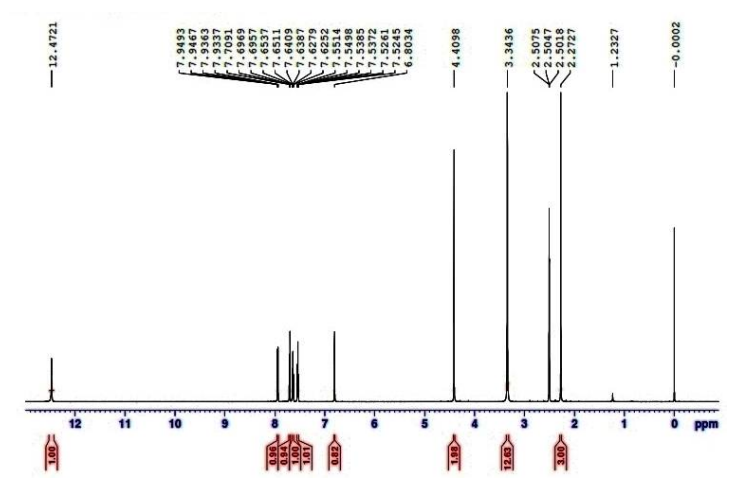

Figure 2: ${ }^{1} \mathrm{H}-\mathrm{NMR}$ spectrum of $8 \mathrm{e}$

$\mathrm{H}-5)$ and 2.27 (s, 3H, $\left.\mathrm{CH}_{3}-6\right)$ while the interconnecting $C$ \& $N$-substituted acetamido moiety in the molecule was inferred by two characteristic peaks at $\delta 12.47(\mathrm{~s}, 1 \mathrm{H}, \mathrm{CON}-\mathrm{H})$, and 4.40 (br.s, $2 \mathrm{H}, \quad \mathrm{CH}_{2}-2^{\prime}$ ). In ${ }^{13} \mathrm{C}-\mathrm{NMR}$ spectrum (Figure 3 ), overall, fourteen signals clearly indicated the successful synthesis of the desired compound. The spectrum identified seven quaternary carbons at $\delta 165.01$ (C-1'), 163.39 (C-5"), 163.30 (C-2"), 156.66 (C-2), 
146.87 (C-4), 131.66 (C-2'"), \& 122.06 (C-1"'), five methines at $\delta 133.30$ (C-4"'), 131.10 (C-6"'), 131.14 (C-3"'), 127.82 (C-5"'), \& 108.12 (C-5), one methylene at $\delta 35.39\left(\mathrm{C}-2^{\prime}\right)$, and a methyl carbon resonating at $\delta 16.84(\mathrm{C}-6)$.

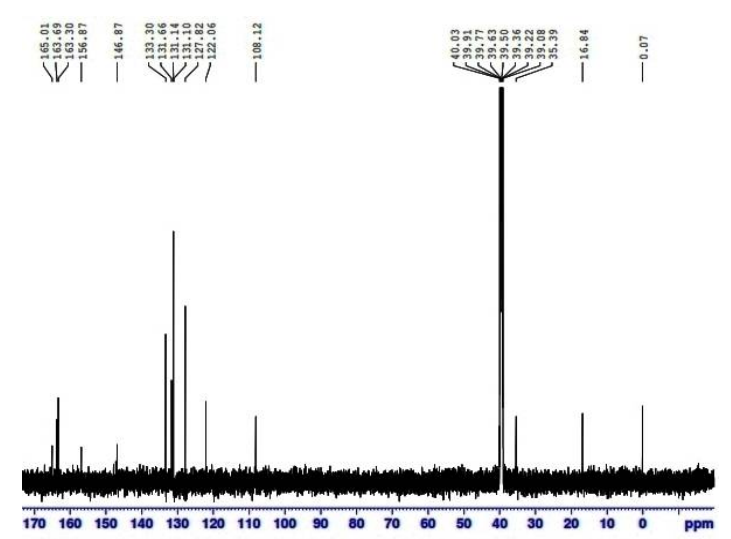

Figure $3:{ }^{13} \mathrm{C}-\mathrm{NMR}$ spectrum of $8 \mathrm{e}$

Various functional groups were ascribed to this molecule by IR data. The prominent absorption bands were observed at $\delta 3350$ ( $\mathrm{N}-\mathrm{H}$ stretching), 2973 (C-H stretching of aromatic ring), 1670 ( $\mathrm{C}=\mathrm{N}$ stretching), 1640 ( $\mathrm{C}=\mathrm{O}$ stretching), 1572 ( $\mathrm{C}=\mathrm{C}$ stretching of aromatic ring), 1159 (C-O-C stretching) and $684\left(\mathrm{C}-\mathrm{Cl}\right.$ stretching $\mathrm{cm}^{-1}$. This corroborated the structural confirmation of $8 \mathbf{e}$ as 2-\{[5-(2-chlorophenyl)-1,3,4-oxadiazol-2-yl] sulfanyl\}- $N$-(4-methyl-1,3-thiazol-2-yl)acetamide. A similar strategy was employed for the structural interpretation of the other compounds in the series.

\section{Enzyme inhibition, molecular docking and structure-activity relationship}

To ascertain the therapeutic potential of 8a-r, these compounds were tested for inhibitory activity against four pharmacologically relevant enzymes, namely, AChE, BChE, a-glucosidase and urease. The results are given as \% inhibition and $\mathrm{IC}_{50}(50 \%$ inhibitory concentration) values in Table 2. The modes of interactions of the synthesized molecules (8a-r) with these enzymes were also described with in silico (molecular docking) study. All compounds except two, 8a and 8r, exhibited an inhibitory potential against AChE. The most promising compounds against this enzyme were $\mathbf{8} \mathbf{I}$ and $\mathbf{8 q}$ with $\mathrm{IC}_{50}$ values of $17.25 \pm 0.07$ and $18.36 \pm 0.08 \mu \mathrm{M}$, respectively, which might have been due to the presence of a substituted nitro group at the three position of the phenyl ring.

Eserine was used as a positive control against this enzyme, having an $\mathrm{IC}_{50}$ of $0.04 \pm 0.0001 \mu \mathrm{M}$. The better activity of $\mathbf{8 I}$ and $\mathbf{8 q}$, relative to other derivatives in the series, might have been due to the presence of a 3-nitrophenyl group and benzyl group, respectively, in these molecules.

Among the mono-chloro substituted analogues, $8 \mathrm{e}-\mathrm{g}$, the order of activity was ortho $>$ meta $>$ para, as per their $\mathrm{IC}_{50}$ values: $8 \mathrm{e}(49.73 \pm 0.15$ $\mu \mathrm{M}), \mathbf{8 f}(53.15 \pm 0.25 \mu \mathrm{M})$, and $\mathbf{8 g}(79.25 \pm 0.05$ $\mu \mathrm{M})$, respectively. It would be expected that the lower the $\mathrm{IC}_{50}$ value, the greater the inhibitory potential of any molecule will be. Dichlorosubstituted $8 \mathrm{~h}(119.53 \pm 0.21 \mu \mathrm{M})$, was less active than the mono-chloroderivatives. Similarly, in the case of amino-substituted analogues, the molecule with meta-amino group, $8 \mathbf{i}(72.85 \pm 0.24$ $\mu \mathrm{M})$, was more active than that with a paraamino group, $8 \mathbf{j}(92.74 \pm 0.15 \mu \mathrm{M})$. For the mononitro regioisomers, $8 \mathbf{k}-\mathbf{m}$, the order of activity was meta $>$ ortho $>$ para as reflected by the $\mathrm{IC}_{50}$ $v$ for $8 \mathrm{l}(17.25 \pm 0.07 \mu \mathrm{M}), 8 \mathbf{k}(29.53 \pm 0.12 \mu \mathrm{M})$, and $8 \mathrm{~m}(36.84 \pm 0.08 \mu \mathrm{M})$, respectively. In the $d i-$ nitro substituted compounds, $\mathbf{8 n - p}$, it was observed that substitution of an additional methyl group rendered the molecule more active relative to substitution with a chloro group. Among these derivatives, the order of activity was: 2-methyl3,5-dinitrophenyl>2-chloro-3,5-dinitrophenyl>3,5dinitrophenyl.This was obvious from their $\mathrm{IC}_{50}$ values of $49.47 \pm 0.13 \mu \mathrm{M}(80), 59.47 \pm 0.25 \mu \mathrm{M}$ $(8 p)$, and $63.80 \pm 0.45 \mu \mathrm{M}(8 \mathbf{n})$.

Varying trends were observed in other derivatives of the series. All derivatives, 8a-r, were docked into the active pocket of this enzyme.

All synthesized derivatives were computationally docked against $\alpha$-glucosidase, AChE and BChE to explore the binding modes of the ligands. Four interactions were shown by the most active compound $\mathbf{8 I}$ in this series. Ser122 makes an acidic interaction with carbonyl oxygen of the ligand, with 8 I giving a bond length of $3.46 \AA$. Trp84 gave the strongest backbone donor interaction with -HNof thiazol and two arenearene interactions with oxadiazol ring, having bond distances of $2.12,3.75$ and $3.93 \AA$, respectively, as identified from the $2 \mathrm{D}$ and $3 \mathrm{D}$ images of Figure 4.

Against BChE, weak activity was shown by the synthesized compounds, and almost half of the synthesized compound were inactive, where a moderate inhibition potential was shown by the compound 8k, with an $\mathrm{IC}_{50}$ of $56.23 \pm 0.09 \mu \mathrm{M}$. This might have been attributed to the substitution of a 2-nitrophenyl group in the skeleton. The docking results revealed that the 8k showed three strong interactions, an acidic interaction between Ser122 and acetyl moiety, a 
backbone donor and an arene-arene interaction with Trp84, having bond lengths of 3.48, 2.07 and $3.86 \AA$, respectively, as indicated in Figure 5 (2D \& 3D).

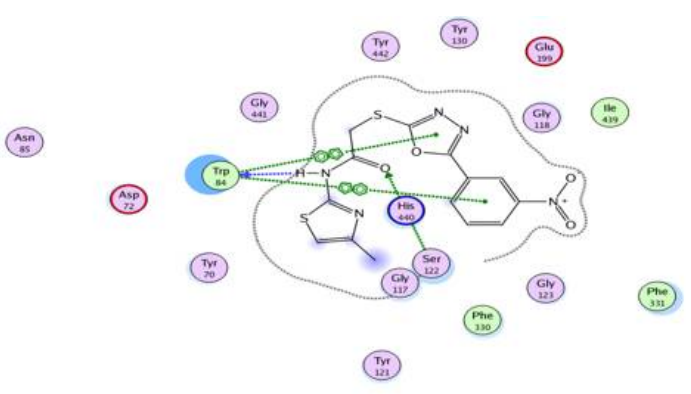

(a) $2 \mathrm{D}$ image

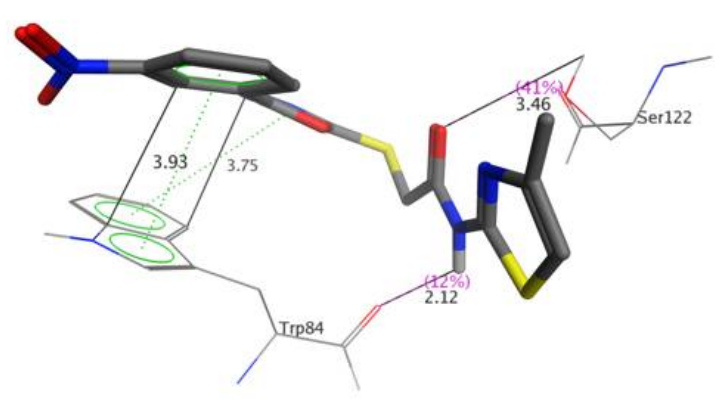

(b) 3D image

Figure 4: The 2D (a) and 3D (b) interaction analysis of 8 I against $A C h E$
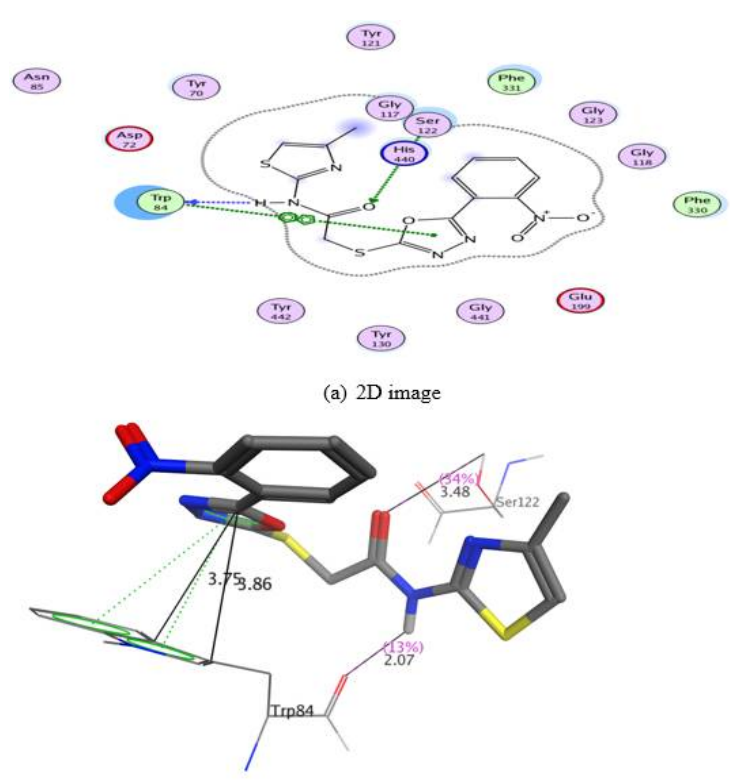

(b) 3D image

Figure 5: The 2D (a) and 3D (b) interaction analysis of 8 k against $\mathrm{BChE}$

Similarly, a very moderate inhibitory potential was demonstrated by most of the compounds against $\alpha$-glucosidase. Acarbose was used as the reference standard $\left(\mathrm{IC}_{50}=37.38 \pm 0.12 \mu \mathrm{M}\right)$. The molecule $80(57.35 \pm 0.17 \mu \mathrm{M})$ exhibited relatively better inhibitory activity in the series studied against this enzyme. This improved activity might have been due to the incorporation of a 2-methyl-3,5-dinitrophenyl ring in the molecule.

The 1,4 glycosidic linkage is hydrolyzed by $\alpha-$ glucosidase. Postprandial hyperglycemia is caused by the inhibition of a-glucosidase [20]. Therefore, inhibition of a-glucosidase is considered important in managing type-2 diabetes whose blood sugar is highest after eating complex carbohydrates. From the in silico study, (Figure 6) the hydrogen attached to nitrogen of the acetamoyl group in 80 was involved in strong polar bonding with Asp349 giving a bond length of $2.12 \AA$. In addition, 1,3,4oxadiazole and 4-methyl-1,3-thiazol-2-yl groups were also found to show arene-cation interactions with Arg312, Arg212 and Arg439, respectively.
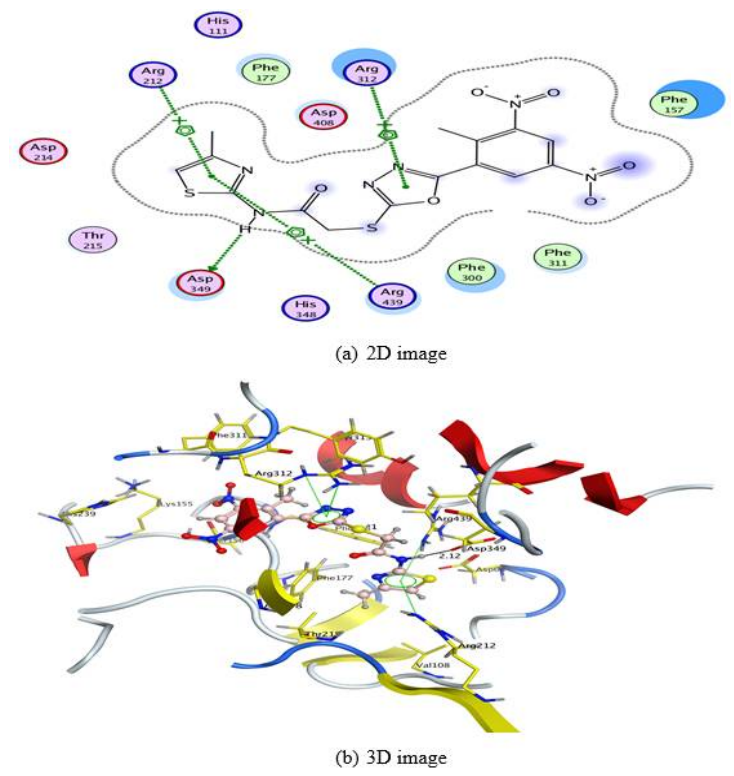

Figure 6: The 2D (a) and 3D (b) interaction analysis of 80 against $\alpha$-glucosidase.

Some compounds were found to be active against urease but displayed feeble inhibition relative to the reference, thiourea $\left(\mathrm{IC}_{50}=\right.$ $21.11 \pm 0.12 \mu \mathrm{M})$. In comparison with the other synthesized derivatives, $8 \mathbf{f}$ possessed relatively considerable inhibitory activity, indicating that the incorporation of 3-chlorophenyl moiety was adequate for the inhibition of this enzyme. Against urease, the molecular docking study (Figure 7) of $\mathbf{8 f}$ revealed $\pi-\pi$ and arene-cation interactions for the 4-methyl-1,3-thiazol-2-yl and 3-chlorophenyl groups with His323 and His324, respectively. The hydrogen attached to nitrogen of the acetamoyl group was involved in polar bonding with Asp224, giving a bond length of $2.16 \AA$.

The cytotoxicity of the synthesized compounds was evaluated by brine shrimp lethality. The 
higher $\mathrm{LD}_{50}$ values of compounds $8 \mathbf{d}$ (498.7 $\mathrm{mM}), 8 \mathrm{l}(466.7 \mathrm{mM})$ and $8 \mathrm{~h}(395.4 \mathrm{mM})$ of brine shrimp lethality analysis demonstrated the lowest toxicity of these compounds. Thus the synthesized molecules might be used as new drug candidates for the related diseases shown by their enzyme inhibition behavior.
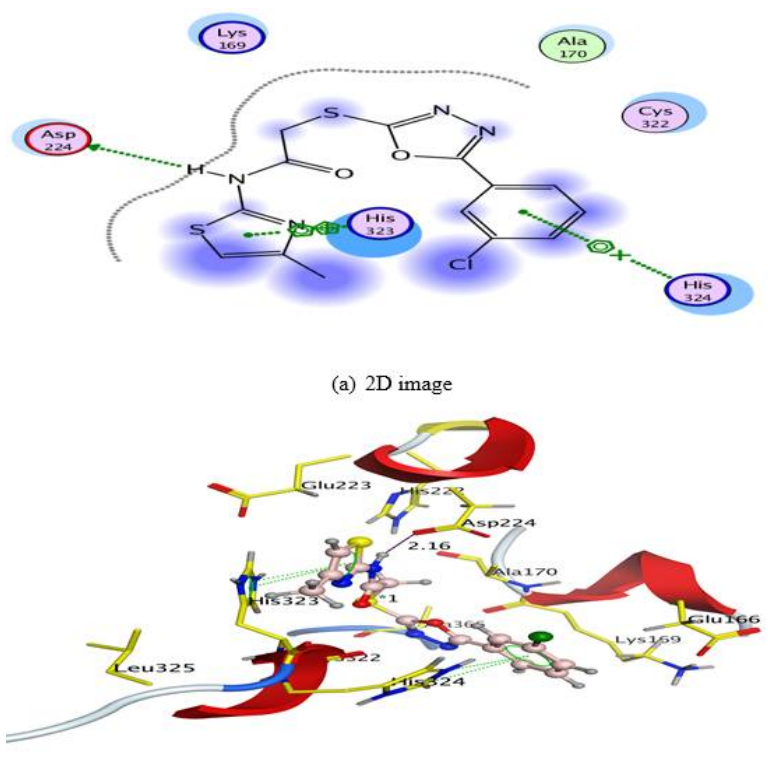

(b) 3D image

Figure 7: The 2D (a) and 3D (b) interaction analysis of $8 f$ against urease.

\section{CONCLUSION}

Most of the derivatives among the synthesized molecules displayed notable inhibitory activity against the selected enzymes, along with very low cytotoxicity. Therefore, these synthesized compounds may have important roles in drug design and development.

\section{DECLARATIONS}

\section{Acknowledgement}

The Higher Education Commission (HEC) of Pakistan is acknowledged by the authors for financial support for the purchase of chemicals and the spectral study. Dr. A. Leyva (USA) provided English editing of the manuscript.

\section{Conflict of Interest}

No conflict of interest associated with this work.

\section{Contribution of Authors}

The authors declare that this work was done by the authors named in this article and all liabilities pertaining to claims relating to the content of this article will be borne by them.

\section{REFERENCES}

1. Siddiqui N, Arshad MF, Ahsan W, Alam MS. Synthesis and fungicidal activity of some novel thiazole Schiff bases derived from benzo-1,3-dioxole. Int J Pharm Sci Drug Res 2009; 1: 136-143.

2. Metzger JV. Thiazole and its derivatives (Part I). An Interscience Publication 1979; 4: 165-167.

3. Boström J, Hogner A, Llinàs A, Wellner E, Plowright AT. Design and synthesis of hydrazine and oxadiazolecontaining derivatives of Sorafenib as antitumor agents. J Med Chem 2011; 55: 1817-1830.

4. Sanchit S, Pandeya SN. Various approaches for synthesis of oxadiazole derivatives. Int $J$ Res Ayurv Pharm 2011; 2: 459-468.

5. Nagaraj T, Chaluvaraju KC, Niranjan MS, Kiran S. 1,3,4Oxadiazole: $A$ potent drug candidate with various pharmacological activities. Int J Pharm Pharm Sci 2012; 3: 9-16.

6. Abbasi MA, Akhtar A, Aziz-ur-Rehmana, Nafeesa $K$, Siddiqui SZ, Khan KM. Synthesis, structural characterization and enzyme inhibition studies on 5-(2nitrostyryl)-1,3,4-oxadiazole-2-thiol derivatives. $J$ Chil Chem Soc 2013; 58: 2186-96.

7. Desai NC, Dodiya AM, Rajpara KM, Rupala YM. Synthesis and antimicrobial screening of 1,3,4oxadiazole and clubbed thiophene derivatives. J Saudi Chem Soc 2014; 18: 255-261.

8. Raval JP, Akhaja TN, Jaspara DM, Myangar KN, Patel $N H$. Synthesis and in vitro antibacterial activity of new oxoethylthio-1,3,4-oxadiazole derivatives. J Saudi Chem Soc 2014; 18: 101-106.

9. Tougu V. Acetylcholinesterase: Mechanism of catalysis and inhibition. Curr Med Chem 2001; 1: 155-170.

10. Gauthier $S$. Cholinergic adverse effects of cholinesterase inhibitors in Alzheimer's disease. Eur $J$ Med Chem 2001; 5: 234-245.

11. Lodhi MA, Abbasi MA, Choudhary MI, Ahmad VU. Kinetics studies on triacontanyl palmitate: a urease inhibitor. Nat Prod Res 2007; 21: 721-725.

12. Lodhi MA, Hussain J, Abbasi MA, Jassbi AR, Choudhary MI. A new Bacillus pasteurii urease inhibitor from Euphorbia decipiens. J Enzyme Inhib Med Chem 2006; 21: 531-535.

13. Kitchen DB, Decornez H, Furr JR, Bajorath J. Docking and scoring in virtual screening for drug discovery: methods and applications. Nat Rev Drug Discov 2004; 3: 935-949.

14. Modi V, Modi P. Oxadiazole: Synthesis, characterization and biological activities. J Saudi Chem Soc 2012; 16 : 327-332.

15. Ellman GL, Courtney KD, Andres V, Featherstone RM. A new and rapid calorimetric determination of acetylcholinesterase activity. Bio Pharm 1961; 7: 88-95. 
16. Pierre C, Roland R, Dube JY. p-Nitrophenol- $\alpha-D$ glucopyranoside as substrate for measurement of maltase activity in human semen. Clin Chem 1978; 24: 208-211.

17. Mobley HL, Cortesia MJ, Rosenthal LE, Jones BD. Characterization of urease from Campylobacter pylori. $J$ Clin Microbiol 1978; 26: 831-836.

18. Wadood A, Riaz M, Jamal SB, Shah M, Lodhi MA. Molecular docking study of $p 4$ benzoxaborol ligands as inhibitor of HCV NS3/4A protease. J Bioinform 2013; 9: 309-314.

19. Ullah N, Bibi G, Kanwal S. Antioxidant and cytotoxic activities and phytochemical analysis of Euphorbia wallichii root extract and its fractions. J Phytochem Anal 2012; 11: 241-249.

20. Harold E, Lebovitz MD. Alpha-glucosidase inhibitors. Endocrinology and Metabolism. Clin North Amer 1997; 26: 539-551. 